\title{
Asset Returns and Financial Fragility
}

\author{
Yang $\mathrm{Li}^{*}$ \\ Department of Economics, Rutgers University \\ ygli@econ.rutgers.edu
}

March 11, 2016

\begin{abstract}
What configuration of asset returns will make the banking system most susceptible to a self-fulfilling run? I study this question in a version of the model of Diamond and Dybvig (1983) with limited commitment and a non-trivial portfolio choice. I show that the relationship between the returns on banks' assets and financial fragility is often non-monotone: a higher return may make banks either more or less susceptible to a run by depositors. The same is true for changes in the liquidation cost and the term premium. I derive precise conditions under which changes in each of these returns increase or decrease financial fragility.
\end{abstract}

Keywords: Financial fragility; Bank runs; Excess liquidity

\footnotetext{
${ }^{*}$ I am grateful to Todd Keister for his infinite support and guidance. I thank Oriol Carbonell-Nicolau, John Landon-Lane, James Peck, Bruno Sultanum for their comments and suggestions, as well as participants at seminar at Rutgers University, especially Hyeon Ok Lee, Yuliyan Mitkov, and Byoung Hark Yoo. All errors remain my own.
} 


\section{Introduction}

How do changes in asset returns affect the susceptibility of the banking system to a run by depositors? In many situations, the answer is not immediately clear. Suppose, for example, that the yield curve becomes steeper, with the return on long-term investments rising relative to short-term rates. Banks would likely respond to this shift by changing the composition of their asset portfolio and the contracts they offer to depositors, and both of these changes will alter the banking system's susceptibility to a run. Higher long-term returns may, for example, lead banks to hold more long-term assets and thus become more illiquid. By itself, this change would tend to make the banking system more fragile. At the same time, however, banks may alter their deposit contracts to provide a higher interest rate for deposits that are held to maturity. By encouraging depositors to not withdraw, this second change would tend to make the banking system more stable. The net effect of a steeper yield curve on banking fragility is thus unclear. Similar competing effects arise in other situations, such as when the short-term rate or the liquidation cost of investment changes.

In this paper, I study how changes in the returns on banks' assets affect financial fragility using a model in the tradition of Diamond and Dybvig (1983). I show that the effect is often non-monotone: a small increase in a particular asset return may increase the susceptibility of banks to a run, but a larger increase may make the banking system more stable. I derive precise conditions under which fragility is increasing or decreasing in each asset return. My analysis is based on a modern version of the Diamond-Dybvig model with the following features. As in Cooper and Ross (1998), there are two assets and banks face a non-trivial portfolio choice. Banks make this choice taking into account the probability of run by depositors, which depends on the realization of a sunspot variable (as in Peck and Shell, 2003, and many others). I also incorporate the limited commitment approach of Ennis and Keister (2009, 2010), which removes the contracting restrictions imposed by Cooper and Ross (1998) while capturing the idea that banks are unable to commit to follow a particular course of action in the event of a crisis. My model is the first to combine a non-trivial portfolio choice with limited commitment and a positive probability of a crisis, which I show generates particularly rich results. ${ }^{1}$

In the environment without commitment, I show that self-fulfilling runs easily emerge as equilibrium outcomes of the model. I also show that, for given parameter values, there exists

\footnotetext{
${ }^{1}$ Cooper and Ross (1998) study a two-asset model with a positive probability of a crisis, but with arbitrary restrictions in the banking contract. Ennis and Keister (2009) study a two-asset model without these restrictions and with limited commitment, but follow Diamond and Dybvig (1983) in assuming that a crisis is an unexpected event. Peck and Shell (2003), Ennis and Keister (2010), Bertolai et al. (2014), Sultanum (2014) and many others study models with varying sources of aggregate uncertainty, but with a single asset and hence no portfolio choice.
} 
a maximum probability with which a run can occur in equilibrium. If the probability of a crisis exceeds this cutoff value, the bank will become sufficiently cautious that running is no longer an equilibrium behavior for depositors. This cutoff value provides a natural measure of financial fragility; if a change in parameter values decreases the maximum probability of a run-equilibrium, I say that it makes the banking system less fragile.

The solution to a typical bank's maximization problem will lie in one of several distinct cases, depending on parameter values and the probability of a crisis. If the probability of a crisis is small, the bank will not hold "excess liquidity", that is, liquid assets that the bank holds over two periods if no crisis occurs. However, it would choose to liquidate its investment in order to meet the withdrawal demand if a crisis does occur. As a crisis becomes more likely, the bank begins to hold excess liquidity to mitigate the liquidation costs. Whenever the probability of a crisis is large enough, the bank becomes even more cautious and holds enough resources as a provision against additional withdrawals that liquidating investment to provide liquidity no longer occurs. I derive the precise conditions under which the bank's best response to depositors' withdrawal decisions lies in each of these cases. I then use this solution to characterize the conditions under which the banking system is fragile in the sense that a bank run equilibrium can arise.

One interesting result that comes out of this analysis is that, under some conditions, the bank will hold excess liquidity as a precaution to mitigate the effects of a potential run. This idea is very natural, but has been surprisingly difficult to capture in the Diamond-Dybvig framework. Cooper and Ross (1998) establish conditions under which a bank would choose to hold excess liquidity. However, as shown by Ennis and Keister (2006), these conditions only apply when the bank is choosing a run-proof contract. In other words, a bank may choose to hold excess liquidity as a way to make itself immune to runs, but in the Cooper-Ross model a bank will never hold excess liquidity for the purpose of mitigating liquidation costs in the event of a run. In my model, this precautionary motive for holding excess liquidity arises naturally.

To study the impact of changes in asset returns on financial fragility, I begin with a simpler case in which there is no liquidation cost for the long-term asset. In this situation, the portfolio choice is trivial and the model effectively reduces to one with a single asset. This approach makes it easier to determine in which case the solution to a bank's maximization problem will lie. This approach also highlights, in as simple a setting as possible, two key elements of my study. First, it enables me to fully understand the relationship between the investment return $R$ and the measure of financial fragility I construct. In particular, I show that the low-return economic system is always stable whenever the fundamental withdrawal demand is sufficiently low. Second, the simpler model is useful for understanding the two 
competing effects that explain why an increase in $R$ can either increase or decrease financial fragility. Such an increase in $R$ does decrease the ex ante incentive for patient depositors to run by providing a higher interest rate for deposits that are held to maturity. However, it also implies that depositors who withdraw in period 1 after it becomes clear that a run has taken place suffer a larger "haircut" relative to depositors who were earlier in the order. This latter effect encourages patient depositors to withdraw early rather than leaving their funds in the banking system, which tends to make the bank more susceptible to a run. I show that either of these two effects can dominate, depending on parameter values.

After establishing these results, I return to the model with liquidation costs and a nontrivial portfolio choice. A unit of good placed into investment in period 0 yields either $R$ in period 2 or $r$ in period 1. I also allow the return on storage to differ across periods, with the return between periods 0 and 1 denoted $\rho_{1}$ and that between periods 1 and 2 denoted $\rho_{2}$. This generalization makes it possible to understand broadly the effects of changes in each of the asset returns $R, r, \rho_{1}$, and $\rho_{2}$ on financial fragility. I first study the case where the coefficient of relative risk aversion $\gamma$ lies in $(1,2]$, in which case I can solve for the equilibrium allocation in closed form. I provide precise conditions under which there is a non-monotone relationship between each asset return and financial fragility. When depositors are more risk averse (i.e. $\gamma>2$ ), closed form solutions are no longer possible, but I provide some limiting results on financial fragility and show that the non-monotone pattern becomes more pronounced with numerical examples. The same principles developed in the special environment with no liquidation cost also apply to this setting. Finally, I study how the changes in the cost of liquidation $\left(\rho_{1}-r\right)$ and the term premium $\left(R-\rho_{1} \rho_{2}\right)$ influence financial fragility. I use the comparative static results described above to show how complex, non-monotone patterns can arise in both cases.

While there has been a surge of interest in models of bank runs and financial fragility in recent years, surprisingly little is known about the comparative statics of these models. Sultanum (2014) characterizes the direct mechanism which implements the constrained efficient outcome in a version of Diamond and Dybvig (1983) with aggregate uncertainty. By varying the realization of the impatient fraction of the population, Sultanum numerically characterizes whether the direct mechanism has a run-equilibrium or not. Bertolai et al. (2014) provide a partial answer to the question of how changes in asset return affect banking system's susceptibility. In an environment with a finite population similar to that in Green and Lin (2003), and the information structure suggested by Peck and Shell (2003), there is a single asset that yields return $R>1$ if held to maturity. They characterize the conditions under which a bank run equilibrium exists for values of $R$ close enough to one and show 
that this equilibrium never exists when $R$ is sufficiently large. ${ }^{2}$ These results seem to suggest that increases in the return on banks' investment promote financial stability. However, they leave open the question of what happens for intermediate values of the return $R$, when it is neither close to one nor very large. Moreover, their one-asset model cannot study effects related to changes in banks' chosen asset portfolios. My paper is the first study to fully characterize the relationship between the returns on banks' assets and financial fragility in the Diamond-Dybvig framework.

The remainder of the paper is organized as follows. In the next section, I present the environment and describe the definitions of financial fragility and stability. In section 3, I analyze equilibrium and characterize the equilibrium measure of financial fragility. I temporarily depart from the assumption that liquidating investment is costly in Section 4, a simplification that allows me to highlight the non-monotonic relationship between the investment return $R$ and the degree of financial fragility. The impact of changes in asset returns on financial fragility in the environment with portfolio choice is introduced in Section 5 and the implications for the effects of liquidation cost and term premium are examined. The paper concludes with Section 6 .

\section{The model}

In this section, I construct a version of the Diamond and Dybvig (1983) model that combines the limited commitment features of Ennis and Keister (2010) with a non-trivial portfolio choice problem as in Cooper and Ross (1998). I begin by describing the physical environment and the basic elements of the model and then define financial fragility and stability in this environment.

\subsection{The environment}

I consider an economy with three periods indexed by $t=0,1,2$. The economy is populated by a $[0,1]$ continuum of ex ante identical depositors, indexed by $i$. I suppose that each depositor has preferences of the form:

$$
u\left(c_{1}, c_{2} ; \omega_{i}\right)=\frac{\left(c_{1}+\omega_{i} c_{2}\right)^{1-\gamma}}{1-\gamma},
$$

where $c_{t}$ represents consumption in period $t=1,2$ and the parameter $\omega_{i}$ is a binomial random variable with support $\Omega \equiv\{0,1\}$. With probability $\pi$ a depositor is impatient (i.e. $\omega_{i}=0$ ) and only values consumption in period 1 ; with probability $1-\pi$ she is patient and values

\footnotetext{
${ }^{2}$ Andolfatto, Nosal and Sultanum (2014) show, using a mechanism design approach in a similar model, that a fairly high asset return between periods 1 and 2 is a sufficient condition for eliminating the possibility of a bank run equilibrium using an indirect mechanism.
} 
the sum of period-1 and period-2 consumption. A depositor's type $\omega_{i}$ (impatient or patient) is private information and is revealed to her at the beginning of period 1 . The fraction of depositors in the population who will be impatient is also $\pi$ due to a law of large numbers. As in Diamond and Dybvig (1983), the coefficient of relative risk-aversion $\gamma$ is assumed to be greater than one. In period 0, depositors are each endowed with one unit of all-purpose good that can be used for consumption or investment. There are two kinds of assets, a short-term, liquid asset and a long-term, illiquid asset. In what follows, I shall refer to them as the short and long assets, respectively. Each asset is represented by a constant-returns-to-scale investment technology. The short asset is represented by a storage technology that allows one unit of the good placed in period 0 to be converted into $\rho_{1}$ units of the good in period 1 ; and one unit of good placed in period 1 yields $\rho_{2}$ units of good in period 2 as in Wallace (1990). The long asset is represented by an investment technology that allows one unit of the good in period 0 to be converted into $R>\rho_{1} \rho_{2}$ units of the good in period 2 . If the long asset is liquidated prematurely in period 1 , it yields $0<r<\rho_{1}$ units of the good for each unit invested.

At the beginning of period 0, depositors pool their resources and set up a bank to insure themselves against individual liquidity risk. The bank then makes a portfolio choice after depositors exit the central location. In period 1, upon learning her preference type, each depositor chooses either to withdraw her funds in period 1 or to wait until period 2. Those depositors who contact the bank in period 1 arrive one at a time in the order given by their index $i$. This index is private information and the bank only observes that a depositor has arrived to withdraw. Under this sequential service constraint, as in Wallace $(1988,1990)$, the bank determines the payment to each withdrawing depositor based on the number of withdrawals that have been made so far. There is no restriction on these payments; the bank can freely choose the amount received by each depositor when she withdraws. Depositors do not observe the bank's portfolio choice or the payments made to other depositors, but they can infer the chosen values in equilibrium. As in Ennis and Keister (2009, 2010), the bank cannot pre-commit to future actions, which implies that the bank must always serve depositors optimally depending on the current situation. The objective of the bank is to maximize welfare measured by the equal-weighted sum of depositors' expected utilities,

$$
\mathcal{W}=\int_{0}^{1} E\left[u\left(c_{1}(i), c_{2}(i) ; \omega_{i}\right)\right] d i
$$

As in Peck and Shell (2003) and others, I introduce an extrinsic "sunspot" signal on which depositors can base their withdrawal decisions. The economy will be in one of two states, $s \in S \equiv\{\alpha, \beta\}$ with probabilities $\{1-q, q\}$. Depositors observe the realization of the state 
of nature at the beginning of period 1. The bank never observes the sunspot state and must infer it based on the observed withdrawal behavior.

\section{$2.2 \quad$ Financial crises and fragility}

After observing her own preference type $\omega_{i}$ and the state $s$, each depositor can choose either to withdraw in period 1 , or to wait until period 2 ,

$$
y_{i}: \Omega \times S \longrightarrow\{0,1\}
$$

where $y_{i}=0$ corresponds to withdrawing at $t=1$ and $y_{i}=1$ corresponds to withdrawing at $t=2$. Let $y$ denote a profile of withdrawal strategies for all depositors. In this game, an equilibrium is a strategy profile for all depositors, together with strategies for the bank, such that every agent is best responding to the strategies of others.

Ennis and Keister (2010) show that this type of game cannot have a full bank run equilibrium. Without loss of generality, I assume a run only occurs in state $\beta$. In order to allow a run to occur with non-trivial probability, I assign the value of $q$ strictly between 0 and 1. ${ }^{3}$ All impatient depositors will clearly choose to withdraw in period 1 , since they receive no utility from consuming in period 2. The interesting question is how patient depositors will behave in state $\beta$. Formally, I study the following partial-run strategy profile for depositors:

$$
\begin{aligned}
& y_{i}\left(\omega_{i}, \alpha\right)=\omega_{i} \quad \text { for all } i, \text { and } \\
& y_{i}\left(\omega_{i}, \beta\right)=\left\{\begin{array}{c}
0 \\
\omega_{i}
\end{array}\right\} \text { for }\left\{\begin{array}{c}
i \leq \pi \\
i>\pi
\end{array}\right\},
\end{aligned}
$$

which corresponds to what Ennis and Keister (2010) call a "one wave" run. Under this specific profile, each patient depositor with $i \leq \pi$ chooses to withdraw early in state $\beta$. Notice that I assume that the remaining patient depositors (those with $i>\pi$ ) do not withdraw early, even if the state is $\beta$ (a crisis is underway), but instead wait and withdraw in period 2. ${ }^{4}$ The following definition provides the notion of financial fragility that I use in the paper.

Definition 1. A banking system is said to be fragile if the strategy profile (1) is part of an equilibrium; otherwise the banking system is said to be stable.

In principle, there are many possible profiles of withdrawal strategies that involve a partial bank run. I show that the same results are obtained if I use a broader definition of fragility in Appendix E.

\footnotetext{
${ }^{3}$ If $q=1$, the bank pays each depositor the value of her initial deposit back and there is again no motivation for a run.

${ }^{4}$ As in Ennis and Keister (2010) a run in this model is necessarily partial.
} 


\section{Equilibrium and financial fragility}

In this section, I first derive the bank's best response to profile (1). I then ask under what conditions the banking system is fragile. Finally, I highlight a key property of the resulting equilibrium allocation: there exists a critical value of $q$ above which the economy is always stable.

\subsection{The best-response allocation}

The bank takes one unit of the good from each depositor in period 0 and invests it in a portfolio consisting of $x$ units of the long asset and $1-x$ units of the short asset. The bank is initially unable to make any inference about the state of nature and chooses to give the same level of consumption $c_{1}$ to each withdrawing depositor with $i \leq \pi$. Once $\pi$ withdrawals have taken place, the bank will be able to infer the state of nature and will use this information to calculate the fraction of its remaining depositors who are impatient, which I denote $\hat{\pi}_{s}$. (Notice that (1) generates $\hat{\pi}_{\alpha}=0$ and $\hat{\pi}_{\beta}=\pi$.) Since all uncertainty has been resolved, the bank will choose to give a common amount $c_{1 s}$ to each (impatient) depositor who withdraws after the fraction of $\pi$ depositors have been served in period 1. In addition, each of the remaining patient depositors will receive a common amount $c_{2 s}$ from the bank's remaining resources when she withdraws in period 2. Given bank's portfolio choice $(1-x, x)$ made in period 0 , these common amounts $c_{1}, c_{1 \beta}, c_{2 \alpha}$, and $c_{2 \beta}$ will be chosen to solve:

$$
\max _{\left\{x, c_{1}, c_{1 \beta}, c_{2 \alpha}, c_{2 \beta}\right\}} \pi u\left(c_{1}\right)+(1-q)(1-\pi) u\left(c_{2 \alpha}\right)+q(1-\pi)\left[\hat{\pi}_{\beta} u\left(c_{1 \beta}\right)+\left(1-\hat{\pi}_{\beta}\right) u\left(c_{2 \beta}\right)\right] .
$$

I can simplify the constraint set for this problem by first noting that it will never be optimal for the bank to liquidate any of the long assets in state $\alpha$. In such a case, the bank could provide more consumption to all depositors by holding more of the short asset and less of the long asset. Similarly, the assumption $R>\rho_{1} \rho_{2}$ implies that it will never be optimal for the bank to hold units of the short asset until $t=2$ in state $\beta$. The bank may, however, hold units of the short asset until $t=2$ in state $\alpha$, and it may choose to meet additional early withdrawal demand by liquidating investment in state $\beta$. Thus, I can write bank's resource constraints as:

$$
\begin{aligned}
\pi c_{1} & \leq \rho_{1}(1-x), \\
(1-\pi) c_{2 \alpha} & =R x+\rho_{2}\left[\rho_{1}(1-x)-\pi c_{1}\right], \\
\rho_{1}(1-x) & \leq \pi c_{1}+(1-\pi) \hat{\pi}_{\beta} c_{1 \beta}, \\
(1-\pi)\left(1-\hat{\pi}_{\beta}\right) c_{2 \beta} & =R\left\{x-\frac{1}{r}\left[\pi c_{1}+(1-\pi) \hat{\pi}_{\beta} c_{1 \beta}-\rho_{1}(1-x)\right]\right\} .
\end{aligned}
$$

The first constraint says that the consumption of the first $\pi$ depositors to withdraw will always come from the resources placed into storage. This constraint may or may not hold 
with equality at the solution. The second constraint says that in state $\alpha$, the remaining patient depositors will consume all of the bank's matured investment plus any resources held in storage for two periods. The third constraint reflects the fact that additional period-1 payments may come only from liquidating investment, since all of the resources in storage have already been depleted. The last constraint is the standard pro rata division of remaining resources that determines the payment in period 2.

The best-response allocation to profile (1) in the two-asset model is summarized by the vector $\mathcal{A}^{*} \equiv\left\{x^{*}, c_{1}^{*}, c_{2 \alpha}^{*}, c_{1 \beta}^{*}, c_{2 \beta}^{*}\right\}$ that solves the problem (2). The explicit derivation of this allocation is given in Appendix A. It is straightforward to show that the solution to this problem will satisfy $c_{1 \beta}^{*}<c_{2 \beta}^{*}$ as long as

$$
\rho_{2} \geq 1
$$

If this inequality were reversed, it could be efficient for some patient depositors to consume in period 1 after the bank has rescheduled payments. Since I am interested in bank runs that lead to inefficient outcomes, in what follows, I shall restrict attention to the case (3).

The above analysis establishes that this solution to the problem (2) will lie in one of the three cases identified in Table 1.

\begin{tabular}{cccc}
\hline & & & State $\alpha$ \\
& & no excess liquidity & \\
State $\beta$ & liquidation & Case I & Case II \\
& no liquidation & & Case III \\
\hline
\end{tabular}

Table 1

In the first case, the bank does not hold excess liquidity for the purpose of providing funds to depositors in the event of a run, and hence it will liquidate investment to provide additional period-1 payments. It is, of course, possible that the bank responds to a run by liquidating investment, even though it holds excess liquidity, which corresponds to the second case. In the third case, the additional early payments come only from the resources in storage without liquidating investment if a crisis occurs. Notice that the bank will never choose to be in the case where there is no excess liquidity and no liquidation. In such a case, the resources in storage have already been paid out to the first $\pi$ depositors who withdrew. Thus, the impatient depositors with $i>\pi$ who have not yet been served would receive no consumption in state $\beta$.

The next result shows when the best-response allocation $\mathcal{A}^{*}$ lies in the different cases in Table 1, depending on the probability of a crisis $q$. For notational convenience, I define the following constants, which depend only on parameter values.

$$
q_{l} \equiv\left\{1+\frac{\rho_{1}-r}{R-\rho_{1} \rho_{2}}\left[\pi \frac{R}{r}+(1-\pi)\left(\frac{R}{r}\right)^{\frac{1}{\gamma}}\right]^{\gamma}\right\}^{-1} \text { and } q_{u} \equiv\left\{1+\frac{\rho_{1}-r}{R-\rho_{1} \rho_{2}}\left[\pi \rho_{2}+(1-\pi)\left(\frac{R}{r}\right)^{\frac{1}{\gamma}}\right]^{\gamma}\right\}^{-1} .
$$


Notice that $0<q_{l}<q_{u}<1$ holds. I then have the following result.

Lemma 1. The bank's best response to profile (1) lies in Case $\left\{\begin{array}{c}\text { I } \\ \text { II } \\ \text { III }\end{array}\right\}$ if $\left\{\begin{array}{c}0<q<q_{l} \\ q_{l} \leq q \leq q_{u} \\ q_{u}<q<1\end{array}\right\}$.

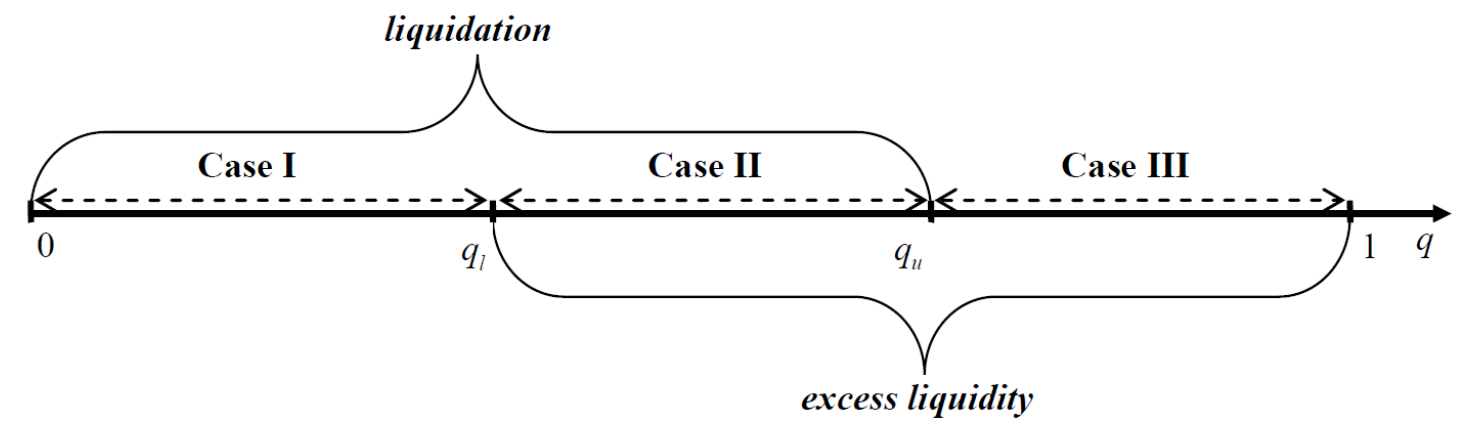

Figure 1: The set of bank's best response to (1)

The intuition for this result is as follows. If a crisis is very unlikely $\left(q<q_{l}\right)$, holding excess liquidity is very costly because of $R>\rho_{1} \rho_{2}$. In this situation, additional period-1 payments will come only from liquidating investment since all of the resources in storage have already been paid out to the first $\pi$ depositors who withdrew. As the probability of a crisis increases, the bank will eventually choose to hold excess liquidity. Having more assets in storage lowers the losses of liquidating investment and thus leaves the bank with more resources in the event of a run. When a crisis is more likely $\left(q>q_{u}\right)$, the bank becomes more cautious and leaves the banking system more liquid, and a bank with a very liquid portfolio will avoid liquidation. Combining these discussions and the above result therefore gives me sufficient conditions under which the best response to (1) involves holding excess liquidity and/or liquidation, as depicted in Figure 1.

\section{$3.2 \quad$ Fragility}

I now verify whether the strategy profile in (1) is part of an equilibrium and hence whether the banking system is fragile or stable. Recall that an impatient depositor will always strictly prefer to withdraw early whatever the payment she receives, since she values period-1 consumption only. Therefore, I only need to consider the actions of patient depositors. Assumption (3) implies $c_{1 \beta}^{*}<c_{2 \beta}^{*}$, and thus a patient depositor with $i>\pi$ prefers to wait in state $\beta$. For patient depositors with $i \leq \pi$, consider separately each of the two possible sunspot states. In state $\alpha$, a patient depositor receives $c_{2 \alpha}^{*}$ if she waits until period 2 , but receives $c_{1}^{*}$ if she withdraws in period 1 . It is straightforward to show that $c_{2 \alpha}^{*}>c_{1}^{*}$ always holds, so that a patient depositor will strictly prefer to wait in state $\alpha$ as specified in (1). In state $\beta$, a patient depositor with $i \leq \pi$ receives $c_{1}^{*}$ if she joins the run and $c_{2 \beta}^{*}$ if she leaves her funds in the banking system. The discussion above establishes that the profile (1) emerges as 
an equilibrium if and only if the allocation $\mathcal{A}^{*}$ satisfies

$$
c_{1}^{*} \geq c_{2 \beta}^{*} .
$$

I now define $\bar{q}$ to be maximum probability with which a run can occur in equilibrium, which is the natural measure of financial fragility in this model.

Definition 2. Given $\left(R, r, \rho_{1}, \rho_{2}, \gamma, \pi\right)$, let $\bar{q}$ be the maximum value of $q$ such that $c_{1}^{*} \geq c_{2 \beta}^{*}$ holds. If $c_{1}^{*} \geq c_{2 \beta}^{*}$ does not hold for any value of $q$, then define $\bar{q}=0$.

In this way, it is possible for me to establish the relationship between asset returns and financial fragility. Most of this paper is concerned with the following question: will an increase in the level of some asset returns would increase or decrease financial fragility? I will first focus on this relationship in a simpler case where the portfolio choice is non-essential, and then I extend the analysis to the full model.

\section{The case with no liquidation cost}

To highlight the relationship between the level of investment return $R$ and the degree of financial fragility, I begin by assuming that there is no liquidation cost, that is, $\rho_{1}=r$; this assumption is subsequently relaxed. ${ }^{5}$ In this special scenario, the portfolio choice becomes trivial because the long asset dominates the short asset in return. By abstracting from the portfolio choice, this environment studied here is analogous to the one-asset Ennis and Keister (2010) model environment with one wave of withdrawals. Considering this limiting case, I show that there exists a non-monotone relationship in the sense that an increase in the return on investment $R$ can either increase or decrease the fragility measure $\bar{q}$. Then I illustrate the competing effects that determine how the return affects financial fragility.

\subsection{The impact of $R$ on fragility}

Bertolai et al.(2014) show in their model that the economy is fragile if the investment return $R$ is close to 1 under some conditions, and that the economy is always stable whenever the return $R$ is sufficiently large. In my model, I establish the relationship between the return on investment and financial fragility more generally and offer the first complete characterization of this comparative static in the Diamond-Dybvig framework. The answer to the question of whether the fragility of an economy increases or decreases when the return $R$ is raised is not obvious because of the competing effects. I first present the fragility measure $\bar{q}$ in this special case with $\rho_{1}=r$, so that the focus is on the remaining asset returns $R$ and $\rho_{1}$.

\footnotetext{
${ }^{5}$ It is worth noting that $q \leq q_{l}=q_{u} \equiv 1$ will necessarily be satisfied if $\rho_{1}=r$. In the case, the bank's best response to (1) always lies in Case I. If there is no term premium (i.e. $R=\rho_{1} \rho_{2}$ ), the bank's best response to (1) only involves Case III, which would lead to exactly the same results in what follows.
} 
When there are relatively few impatient depositors, there will be sufficient assets left for the bank to offer a relatively high payment to patient depositors who wait until period 2 , which implies that bank runs never occur in equilibrium. In other words, when $\pi$ is low, the economy is stable for all $q$. In particular, define

$$
\pi_{F} \equiv \frac{\left(\frac{R}{\rho_{1}}\right)^{\frac{1}{\gamma}}-1}{\left(\frac{R}{\rho_{1}}\right)^{1-\frac{1}{\gamma}}-1} .
$$

Note that $0<\pi_{F}<1$ holds whenever $\gamma>2 .{ }^{6}$ I then have the following result.

Proposition 1. Suppose $\rho_{1}=r$. If $\pi>\pi_{F}$, then

$$
\bar{q}=\frac{\frac{\rho_{1}}{R}-\left[\pi\left(\frac{R}{\rho_{1}}\right)^{1-\frac{1}{\gamma}}+(1-\pi)\right]^{-\gamma}}{1-\left[\pi\left(\frac{R}{\rho_{1}}\right)^{1-\frac{1}{\gamma}}+(1-\pi)\right]^{-\gamma}} ;
$$

otherwise, $\bar{q}=0$.

When $\pi$ is large, more depositors have a real need to consume early. As a result, when a run occurs, the bank will realize a crisis is occurring relatively late. After a large number of depositors have been served, the remaining resources are relatively small, which would lead the bank to optimally provide smaller payments to patient depositors who withdraw in period 2. Thus, the banking system tends to be fragile. However, when $\pi$ is small enough, the patient depositors prefer to leave their deposits in the banking system because the bank has sufficient resources to offer after a small amount of early withdrawals have been served.

Proposition 1 implies that when both $\pi$ and $R$ are small enough, the economy would be stable (i.e. $\bar{q}=0$ ). This result is in sharp contrast to the property in Bertolai et al. (2014), which shows that the economy tends to be fragile as $R$ is close to 1 under some conditions.

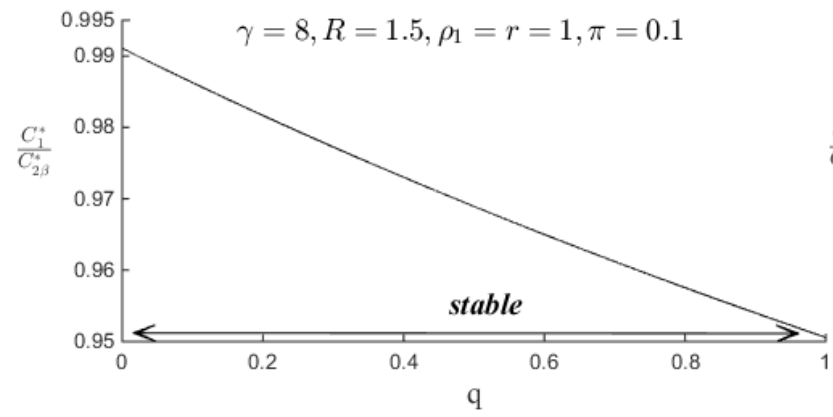

(a) $\pi<\pi_{F}$

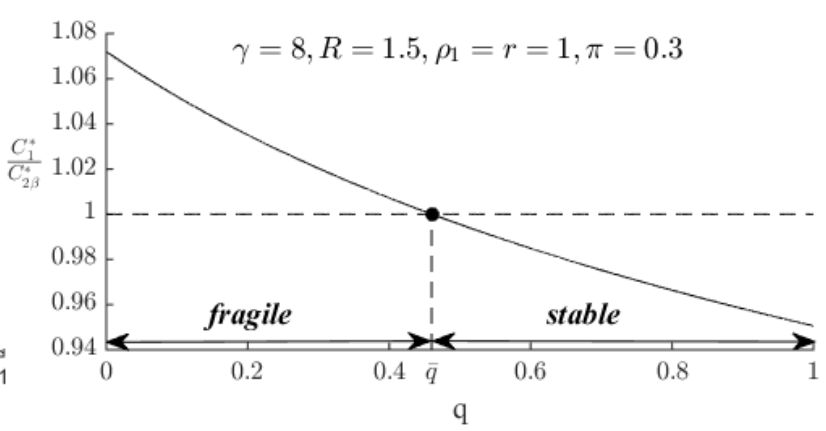

(b) $\pi>\pi_{F}$

Figure 2: $c_{1}^{*} / c_{2 \beta}^{*}$ with $\pi$ varied in the environment with no liquidation cost

Figure 2 plots the ratio $\frac{c_{1}^{*}}{c_{2 \beta}^{*}}$ as a function of the probability of state $\beta$. In the left panel, $\pi$ is relative small and, as result, this ratio is always smaller than one; the economy is stable for

\footnotetext{
${ }^{6}$ It is straightforward to show that the economy with no liquidation cost is always stable whenever $1<\gamma \leq 2$.
} 
all values of $q$. In the right panel, in contrast, the economy is fragile when $q$ is small enough. The figure shows how $\bar{q}$ is determined as the point where the ratio $\frac{c_{1}^{*}}{c_{2 \beta}^{*}}$ crosses 1 .

I now turn to my primary interest: determining how the changes in $R$ influence $\bar{q} .^{7}$ Suppose there is an increase in the investment return $R$. Would financial fragility tend to increase or decrease? I define

$$
\tilde{\pi} \equiv \frac{2-\frac{1}{\gamma}}{\gamma-\frac{1}{\gamma}}
$$

I then have the following result.

Proposition 2. Suppose $\rho_{1}=r$ and $\pi>\pi_{F}$. If:

- $\pi>\tilde{\pi}$, then $\bar{q}$ is strictly decreasing in $R$;

- $\pi<\tilde{\pi}$, then there exists $\tilde{R}>\rho_{1}$ such that $\bar{q}$ is strictly $\left(\begin{array}{c}\text { increasing } \\ \text { decreasing }\end{array}\right)$ in $R$ as $R\left(\begin{array}{c}< \\ >\end{array}\right) \tilde{R}$.

This proposition establishes precise conditions under which there is a non-monotone relationship between the return $R$ and financial fragility $\bar{q}$, as illustrated in Figure 3.

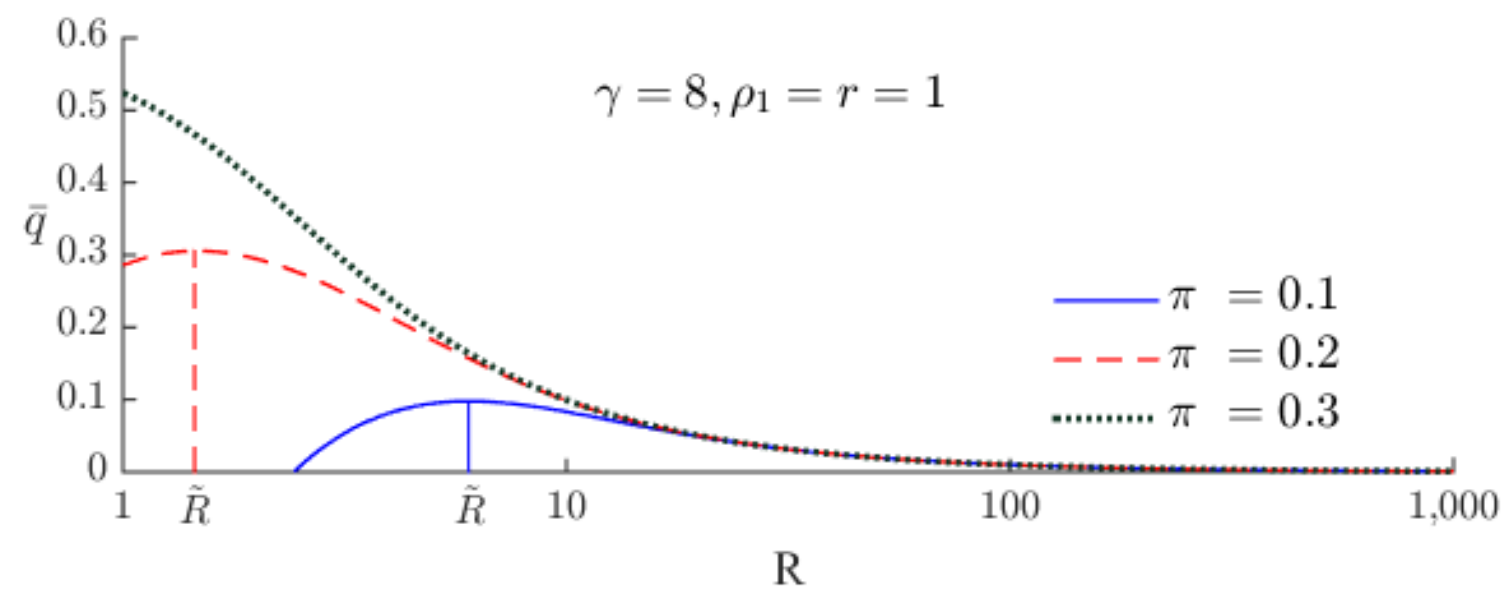

Figure 3: The measure of fragility in the environment with no liquidation cost

Figure 3 depicts $\bar{q}$ as a function of $R$ for three different values of $\pi$ (note the logarithmic scale for the x-axis). Notice that an increase in $R$ will make the economy less susceptible to a run if the return on investment is high enough. In some cases, however, (for example when $\pi$ equals 0.1 or 0.2 and $R$ is moderate), the increase in $R$ can either increase or decrease financial fragility, depending on parameter values.

\subsection{Competing effects on fragility}

To understand intuitively the non-monotone pattern in Figure 3 , it helps to write $c_{1}^{*} / c_{2 \beta}^{*}$ as

$$
\frac{c_{1}^{*}}{c_{2 \beta}^{*}} \equiv \frac{c_{1 \beta}^{*}}{c_{2 \beta}^{*}} \times \frac{c_{1}^{*}}{c_{1 \beta}^{*}} .
$$

${ }^{7}$ Note that asset returns appear as a form of $\frac{R}{\rho_{1}}$ in $\bar{q}$. Without loss of generality, I focus on the analysis of $R$. Focusing on $\rho_{1}$ would exactly lead to the reversed result. 
The following proposition explains that the change in $R$ has a basic trade-off between "period" substitution effect and "state" substitution effect in the environment with no liquidation cost. Proposition 3. If $\rho_{1}=r$, then $\left(\begin{array}{c}c_{1 \beta}^{*} / c_{2 \beta}^{*} \\ c_{1}^{*} / c_{1 \beta}^{*}\end{array}\right)$ is strictly $\left(\begin{array}{c}\text { decreasing } \\ \text { increasing }\end{array}\right)$ in $R$.

Suppose that I increase $R$, holding other parameter values fixed. Such an increase leads the bank to raise $c_{2 \beta}^{*}$ relative to $c_{1 \beta}^{*}$ because providing consumption in period 2 is now relatively less expensive. This fact deceases the ex ante incentive for depositors to run. At the same time, in contrast, the bank tends to raise $c_{1}^{*}$ relative to $c_{1 \beta}^{*}$ because providing consumption in state $\alpha$ is now relatively less expensive compared to the state $\beta$. By itself, this change increases the incentive for a depositor who is early in the order to join the run and collect the payment $c_{1}^{*}$, before the bank learns the state. As a result, the latter effect encourages patient depositors to withdraw early rather than leaving their deposits in the banking system, which tends to make the bank more susceptible to a run.

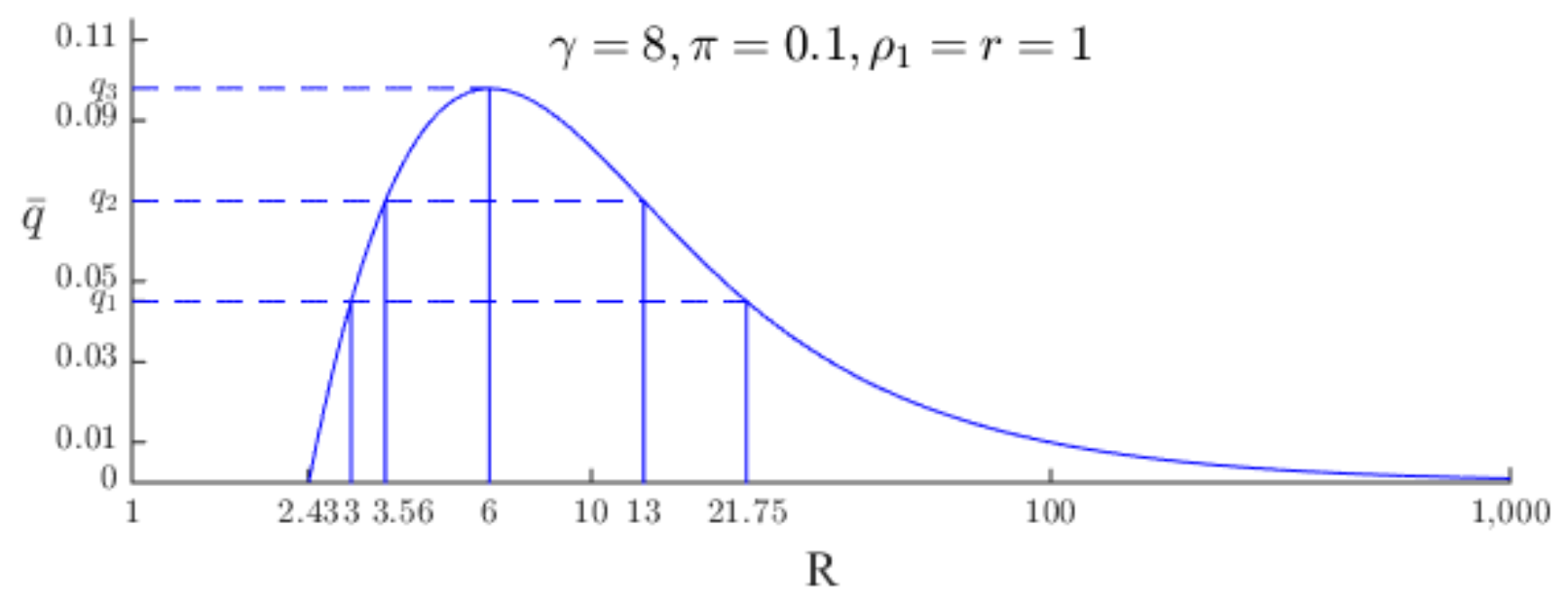

(a) The impact of $R$ on $\bar{q}$

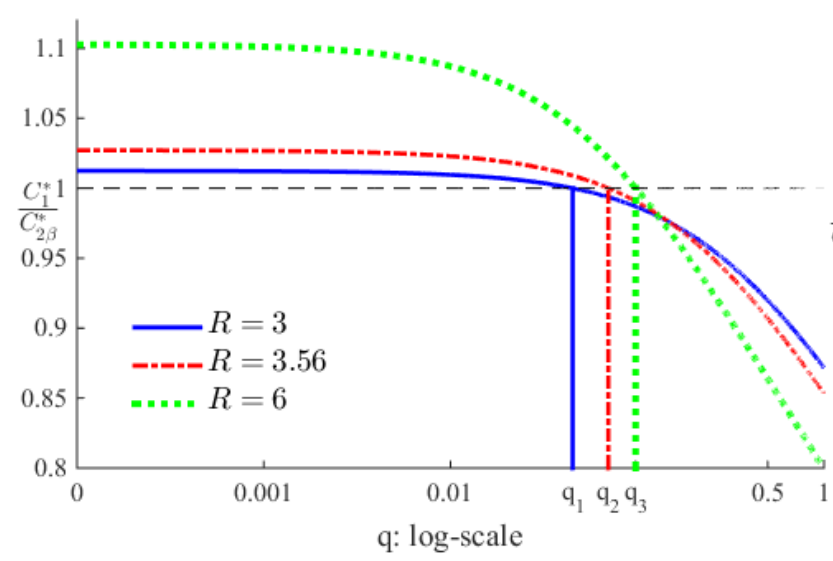

(b) $\bar{q}$ is increasing in $R$

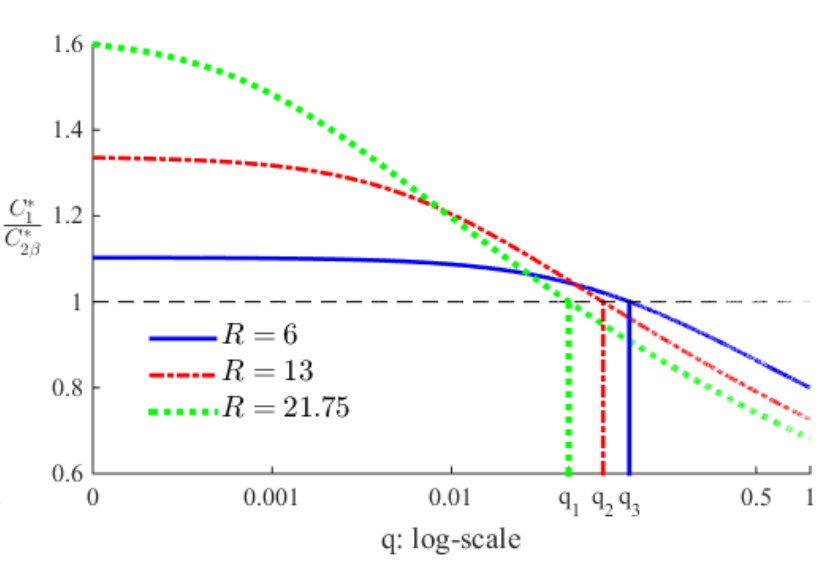

(c) $\bar{q}$ is decreasing in $R$

Figure 4: Two competing effects in the environment with no liquidation cost 
The top panel in Figure 4 repeats the solid blue curve from Figure 3, but with an expanded scale on the vertical axis. The bottom two panels show that either of these two effects can dominate, as stated in Proposition 3. To understand the non-monotonic pattern, I ask the following question. What is the maximum value of $q$ satisfying $\frac{c_{1}^{*}}{c_{2 \beta}^{*}} \geq 1$ for a given asset return $R$ ? As the last two panels illustrate, it is evident that the ratio $\frac{c_{1}^{*}}{c_{2 \beta}^{*}}$ is strictly decreasing in $q$. Thus, I can determine whether there exists a cutoff value $\bar{q}$ such that the ratio $\frac{c_{1}^{*}}{c_{2 \beta}^{*}} \geq(<) 1$ if $q \leq(>) \bar{q}$, for any given values of $R$. Panel (b) shows that, for a given value of $q<q_{3}$, the "state" substitution effect is dominant and an increase in $R$ will increase the ratio $\frac{c_{1}^{*}}{c_{2 \beta}^{*}}$ when $R$ is small. This rise, in turn, increases the threshold value of $q$ from $q_{1}$ to $q_{3}$. Panel (c) illustrates that as the investment return increases further, however, for a given value of $q>q_{1}$, the "period" substitution effect outweighs the "state" substitution effect. In this

situation, an increase in $R$ will decrease the ratio $\frac{c_{1}^{*}}{c_{2 \beta}^{*}}$, which translates into a decline in the threshold value of $q$ from $q_{3}$ to $q_{1}$.

In the next section, I turn to the study of how the measure of fragility varies with comparative statics of $R, r, \rho_{1}$, and $\rho_{2}$ in the presence of an essential portfolio choice.

\section{The full model}

The model of intermediation presented above is, in some ways, rather special: there is no role for excess liquidity in an environment with no portfolio choice. While these features were useful for generating intuition, they are by no means necessary for the non-monotone pattern reported in Proposition 2 to obtain. I now demonstrate this fact by removing the assumption of no liquidation cost. The general logic of my analysis here is parallel to that just used. I first identify how changes in asset returns influence financial fragility in this economy assuming that liquidation is costly. Then I illustrate the two competing effects of an increase in asset returns under different cases in the light of Lemma 1.

\subsection{A general fragility result}

With an essential portfolio choice in this environment, the structure of equilibrium outcomes is more complex than before. Using Appendix A, the measure of financial fragility can be formalized in a general formula. I define

$$
\begin{aligned}
f(\cdot) & =\left[\pi\left(\frac{R}{r}\right)^{1-\frac{1}{\gamma}}+(1-\pi)\right]^{\gamma}-\frac{R}{\rho_{1}} \\
g(\cdot) & =\left[\pi\left(\frac{R}{r}\right)^{1-\frac{1}{\gamma}}+(1-\pi)\right]^{\gamma}-\frac{R^{2}-\left(\rho_{2}+1\right) r R+\rho_{1} \rho_{2} r}{R\left(\rho_{1}-r\right)} \\
h(q) & =\pi \rho_{2}\left(\rho_{2}+\frac{1-\frac{\rho_{1} \rho_{2}}{R}}{q}\right)^{-\frac{1}{\gamma}}+(1-\pi)-\left[\frac{(1-q) \frac{R}{\rho_{1}}}{1-q \frac{R}{\rho_{1}}}\right]^{\frac{1}{\gamma}} .
\end{aligned}
$$


Proposition 4. Given $R, r, \rho_{1}, \rho_{2}$ and $\gamma$,

- if $f(\cdot) \leq 0$, then the economy is stable for all $q$ and, therefore, $\bar{q}=0$;

- if $f(\cdot)>0$ and $g(\cdot) \leq 0$, then

$$
\bar{q}=\frac{\frac{\rho_{1}}{R}-\left[\pi\left(\frac{R}{r}\right)^{1-\frac{1}{\gamma}}+(1-\pi)\right]^{-\gamma}}{1-\left[\pi\left(\frac{R}{r}\right)^{1-\frac{1}{\gamma}}+(1-\pi)\right]^{-\gamma}} \equiv \bar{q}_{\text {Case I }} ;
$$

- if $g(\cdot)>0$ and $\left\{q \mid h(q)=0 ; q \in\left(q_{u}, 1\right)\right\}=\emptyset$, then

$$
\bar{q}=\frac{\left(R-\rho_{1} \rho_{2}\right) r}{\left(R-\rho_{2} r\right) R} \equiv \bar{q}_{\text {Case II }} ;
$$

- if $g(\cdot)>0$ and $\left\{q \mid h(q)=0 ; q \in\left(q_{u}, 1\right)\right\}=\mathcal{Q}\left\{q_{1}, q_{2}, \ldots, q_{n}\right\}$, then

$$
\bar{q}=q_{m} \equiv \bar{q}_{\text {Case III }} \text {, where } q_{m} \text { is the biggest element of } \mathcal{Q} \text {. }
$$

Lemma 1 shows that the bank's best response lies in Case I, Case II, and Case III respectively as $q$ increases, and hence there might exist different explicit conditions under which the economy is fragile.

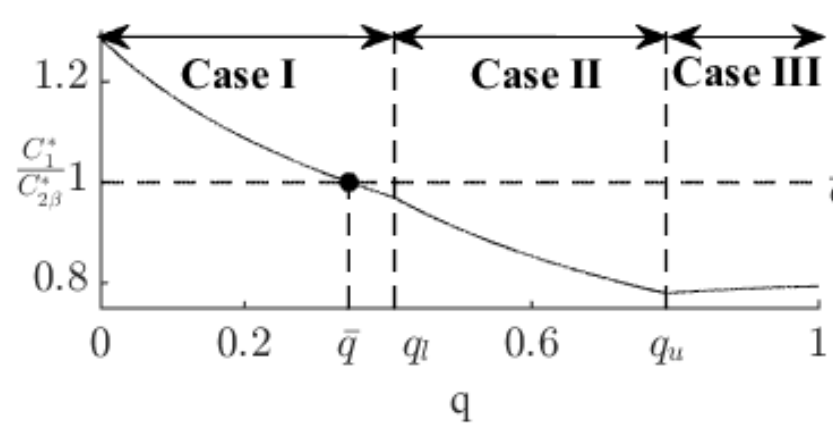

(a) $R=2.5: \bar{q}$ in Case I

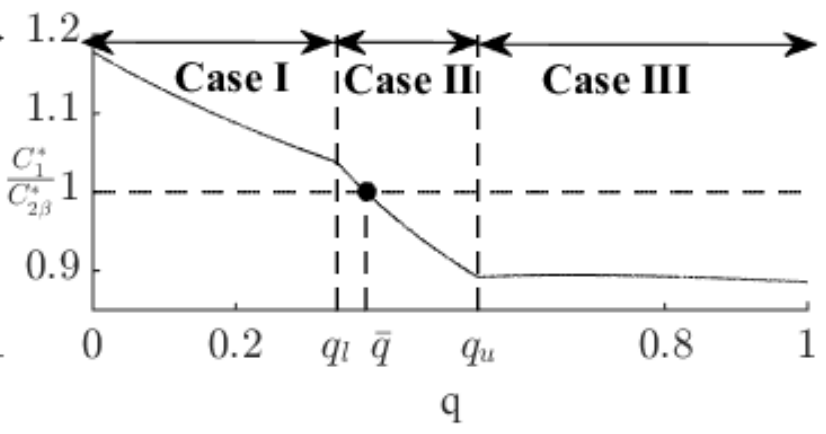

(b) $R=1.8: \bar{q}$ in Case II

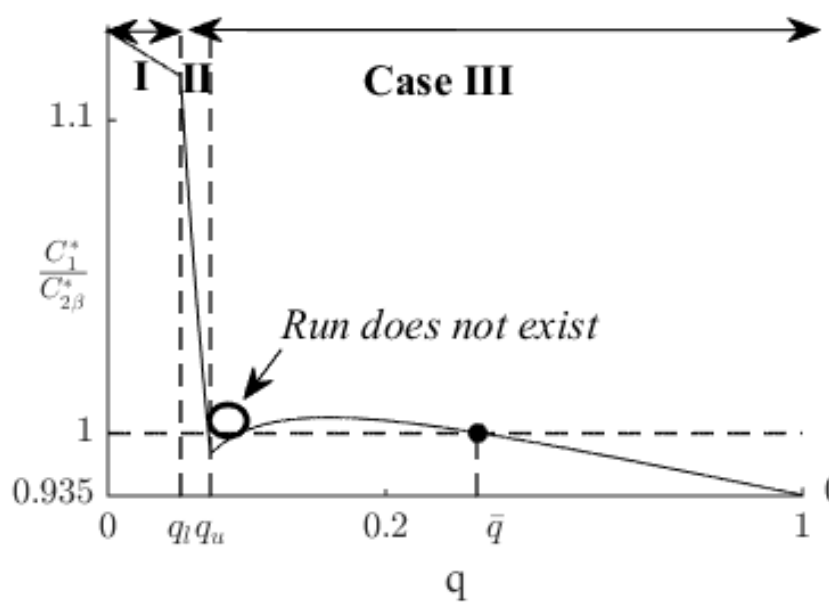

(c) $R=1.52: \bar{q}$ in Case III

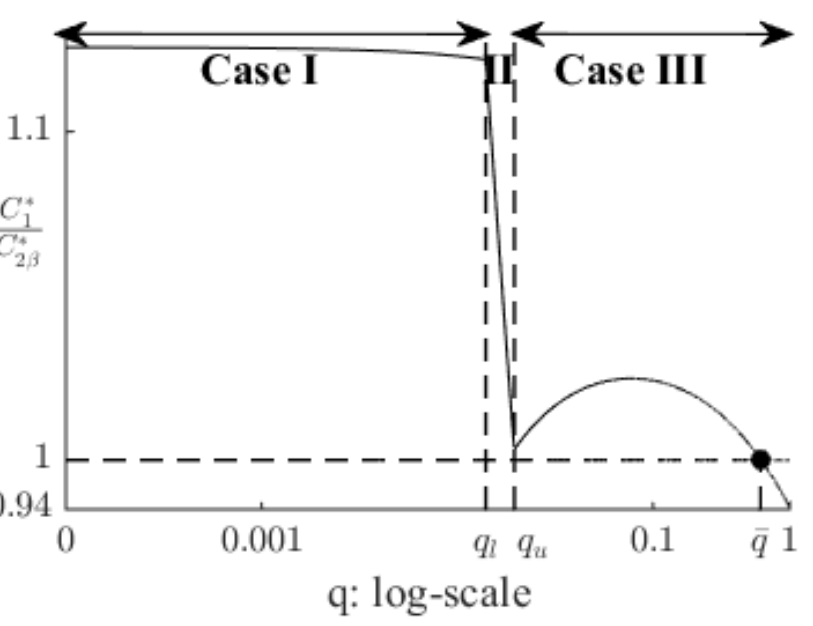

(d) $R=1.505: \bar{q}$ in Case III

Figure 5: $\frac{c_{1}^{*}}{c_{2 \beta}^{*}}$ in the economy $\left(\gamma=3, \pi=0.85, r=1.1, \rho_{1}=1.25, \rho_{2}=1.2\right)$ with $R$ varied 
It is straightforward to show that $\frac{c_{1}^{*}}{c_{2 \beta}^{*}}$ is strictly decreasing in $q$ because of costly liquidation whenever the bank's best response lies in Case I or Case II (i.e. $q \leq q_{u}$ ). In such a situation, there exists a threshold value of $q$ below which fragility arises. Things are different when the bank's best response lies in Case III (i.e. $q>q_{u}$ ). In this case, the bank becomes more cautious and holds sufficient short assets in its portfolio so that it avoids the loss of liquidating investment. Figure 5 illustrates that an increase in the probability of a crisis can either increase or decrease the ratio $\frac{c_{1}^{*}}{c_{2 \beta}^{*}}$ under Case III, depending on parameter values. ${ }^{8}$ Moreover, panel (c) of Figure 5 shows that the run equilibrium does not exist for some values of $q$ less than $\bar{q} \cdot{ }^{9}$

As an aside, note that the bank does choose to hold excess liquidity in some cases. Cooper and Ross (1998) analyze the question of when is excess liquidity held. Ennis and Keister (2006) show that - contrary to what Cooper and Ross (1998) suggest - the bank will never hold excess liquidity for the purpose of meeting early withdrawal demand in the event of a run in their model. However, my study here captures the idea of Cooper and Ross (1998) aimed to show: holding excess liquidity to mitigate the loss of liquidating investment is attractive in some situations. This fact can be seen in Figure 5 in which $\bar{q}$ lies in either Case II or Case III.

\subsection{Comparative statics when $1<\gamma \leq 2$}

Would the pattern of $\bar{q}$ as a function of asset returns be non-monotonic as in the environment with no liquidation cost? Do there exist two competing effects on fragility? I first study the case $1<\gamma \leq 2$ because I can derive the explicit expression for $\frac{c_{1}^{*}}{c_{2 \beta}^{*}}$ as a function of each asset return. ${ }^{10}$ Therefore, starting with this case provides a clear picture of the impact of asset returns on financial fragility, but also is useful for providing critical insights into the more complex cases with $\gamma>2$.

In keeping with my earlier analysis, I begin by providing precise conditions under which $\bar{q}$ is increasing or decreasing in $R$. It is important to bear in mind that the economy is always stable whenever $f(\cdot) \leq 0$ holds, so I restrict attention to the case of $f(\cdot)>0$ through out the remaining study. In addition, the degree of financial fragility $\bar{q}$ will be given by (7) or (8) depending on the sign of function $g(\cdot)$. The following proposition characterizes the outcomes in this case. I define the critical values $\underline{R}$ and $R^{*}$ by

$$
\underline{R}=\rho_{1} \rho_{2}\left(1+\sqrt{1-r / \rho_{1}}\right) \text { and } g\left(R^{*}\right)=0 .
$$

\footnotetext{
${ }^{8}$ This fact is similar in spirit to Proposition 5 in the Ennis and Keister (2006). In addition, the probability of a crisis can affect the level of investment in a relatively intricate way.

${ }^{9}$ Appendix $\mathrm{C}$ repeats panel (c) with an expanded scale on the vertical axis.

${ }^{10}$ In light of Proposition 4, it is straightforward to show that there is a cutoff value of $q$ below which the economy is fragile and above which it is always stable, given $R, r, \rho_{1}, \rho_{2}, \pi$, and $1<\gamma \leq 2$ with $f(\cdot)>0$. Moreover, in this case the equilibrium outcome will never fall in Case III since $\{q \mid h(q)=0 ; q \in(q u, 1)\}=\emptyset$ always holds.
} 
Proposition 5. Assume $f(\cdot)>0$ holds. For all $\gamma \in(1,2]$, $\bar{q}$ is strictly $\left\{\begin{array}{ll}\left(\begin{array}{c}\text { increasing } \\ \text { decreasing }\end{array}\right) \text { in } R \text { with } R\left(\begin{array}{c}< \\ >\end{array}\right) \min \left\{R^{*}, \underline{R}\right\}, & \text { if } \pi<\left(\frac{r}{\rho_{1}-r}\right)^{\frac{1}{\gamma}} \cdot \mathbb{1}\{\gamma=2\} \\ \left(\begin{array}{c}\text { increasing } \\ \text { decreasing }\end{array}\right) \text { in } R \text { with } R\left(\begin{array}{c}< \\ >\end{array}\right) \underline{R}, \quad \text { if } \pi>\left(\frac{r}{\rho_{1}-r}\right)^{\frac{1}{\gamma}} \cdot \mathbb{1}\{\gamma=2\}\end{array}\right\}$,

where $\mathbb{1}\{\gamma=2\}$ is an indicator function such that $\mathbb{1}\{\gamma=2\}=1$ if $\gamma=2$ and 0 otherwise.

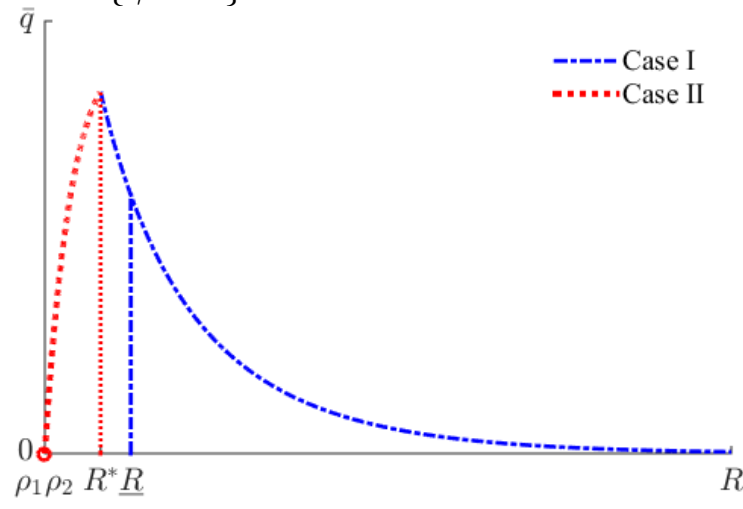

(a) $\pi<\left(\frac{r}{\rho_{1}-r}\right)^{\frac{1}{\gamma} \cdot \mathbb{1}\{\gamma=2\}}$

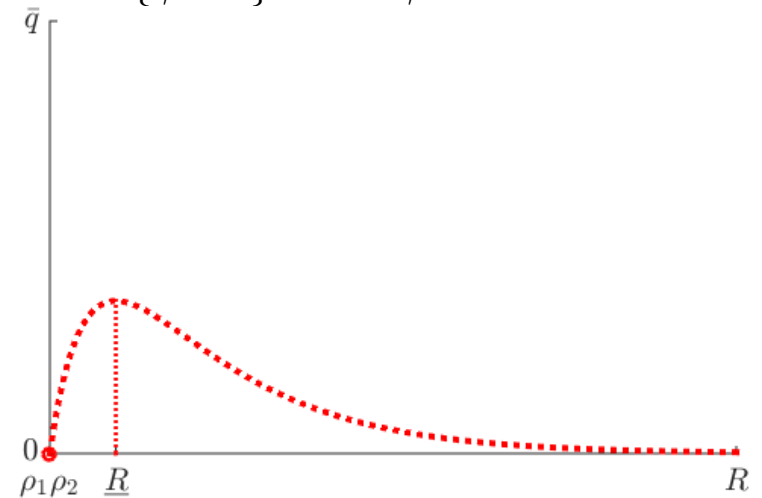

(b) $\pi>\left(\frac{r}{\rho_{1}-r}\right)^{\frac{1}{\gamma} \cdot \mathbb{1}\{\gamma=2\}}$

Figure 6: The impact of $R$ on $\bar{q}$ with $1<\gamma \leq 2$

The blue dashed line in Figure 6 corresponds to the threshold value of $q$ when the bestresponse allocation lies in Case I. Similarly, the red dotted line represents the threshold value of $q$ in Case II. It is worth noting that the extreme economy of $R=\rho_{1} \rho_{2}$ is always stable, which is depicted by an empty circle. ${ }^{11}$

Two observations immediately follow. Firstly, the right panel of Figure 6 shows that the degree of financial fragility $\bar{q}$ as a function of $R$ can have a non-monotone pattern within the Case II. Secondly, the non-monotonicity in the two-asset model can also stem from the fact that there might be different effects on $\bar{q}$ by varying $R$ in Case I and Case II. For example, the first panel of Figure 6 shows that a higher $R$ reduces financial fragility while the economy is in Case I, but it increases instability in Case II. In this case, the change in the bank's best response regions for $\bar{q}$ generates the non-monotonicity. Hence, I conclude here that the non-monotonicity result reported in Proposition 2 is robust to the environment with portfolio choice.

Recall that there are two competing effects - "period" substitution effect and "state" substitution effect demonstrated by equation (6) in Section 4.2. In Case I, a higher $R$ increases the payment to patient depositors who withdraw in period 2 in state $\beta$, which reduces the incentives for patient depositors to join the run. In the meantime, the bank offers

\footnotetext{
${ }^{11}$ This fact can be easily verified by looking at the condition $\pi \leq \pi_{F}$.
} 
a higher payment to depositors who withdrew before it observes the state of nature. Since the coefficient of relative risk aversion $\gamma \leq 2$ in this subsection, the "period" substitution effect is dominant and thus $c_{1}^{*} / c_{2 \beta}^{*}$ is decreasing in $R$ in Case I. Recall that the ratio $c_{1}^{*} / c_{2 \beta}^{*}$ decreases as the probability of a crisis $q$ rises in Case I. Therefore, a higher $R$ reduces the fragility of an economy in Case I. When the equilibrium outcome falls in Case II, the "state" substitution is dominant as $R$ is close to $\rho_{1} \rho_{2}$, which implies that $c_{1}^{*} / c_{2 \beta}^{*}$ is increasing in $R$. As $R$ increases further, in some economies, however, eventually the "period" substitution effect will dominate the "state" substitution effect because providing consumption in period 2 is now less expensive.

Similarly, the following proposition establishes precise conditions under which $\bar{q}$ is increasing or decreasing in other asset returns. I define the critical values $\pi^{*}, r^{*}, \rho_{1}^{*}$ and $\rho_{2}^{*}$ by

$$
\pi^{*}=\frac{\left[\frac{R^{2}-2 r R+\rho_{1} r}{R\left(\rho_{1}-r\right)}\right]^{\frac{1}{\gamma}}-1}{\left(\frac{R}{r}\right)^{1-\frac{1}{\gamma}}-1}, g\left(r^{*}\right)=0, g\left(\rho_{1}^{*}\right)=0, \text { and } g\left(\rho_{2}^{*}\right)=0 .
$$

I then have the following result.

Proposition 6. Assume $f(\cdot)>0$ holds. For all $\gamma \in(1,2]$,

- $\bar{q}$ is strictly $\left\{\begin{array}{l}\text { increasing } \\ \text { decreasing }\end{array}\right\}$ in $r$ with $r\left\{\begin{array}{c}< \\ >\end{array}\right\} r^{*}$

- $\bar{q}$ is strictly $\left\{\begin{array}{l}\text { increasing } \\ \text { decreasing }\end{array}\right\}$ in $\rho_{1}$ with $\rho_{1}\left\{\begin{array}{l}< \\ >\end{array}\right\} \rho_{1}^{*}$

- $\bar{q}$ is $\left\{\begin{array}{lll}\left(\begin{array}{c}\text { constant } \\ \text { decreasing }\end{array}\right) \text { in } \rho_{2} \quad \text { with } \rho_{2}\left(\begin{array}{l}< \\ >\end{array}\right) \rho_{2}^{*}, & \text { if } \pi<\pi^{*} \\ \text { decreasing } & \text { in } \rho_{2}, & \text { if } \pi>\pi^{*}\end{array}\right\}$

This proposition shows that there also can exist a non-monotone effect of the returns $r$, $\rho_{1}$, and $\rho_{2}$ on financial fragility.

\subsection{Comparative statics when $\gamma>2$}

I now turn to the case where the coefficient of relative risk aversion is larger than 2. The results in this scenario are more complex because the run-equilibrium outcome will sometimes fall in Case III. An increase in the probability of a crisis can either increase or decrease the ratio $\frac{c_{1}^{*}}{c_{2 \beta}^{*}}$ under Case III as illustrated in Figure 5. Appendix D demonstrates that, when the two-asset model is formalized in the environment with $\gamma>2$, the non-monotonicity with respect to the impact of changes in asset returns on financial fragility becomes more 
pronounced at least in the neighborhood of some limiting values. ${ }^{12}$

Figure 7 provides a series of illustrative examples with respect to the impact of $R$ on $\bar{q}$. The red and blue curves represent the same cases as in Figure 6, but the green solid line corresponds to $\bar{q}$ in Case III.

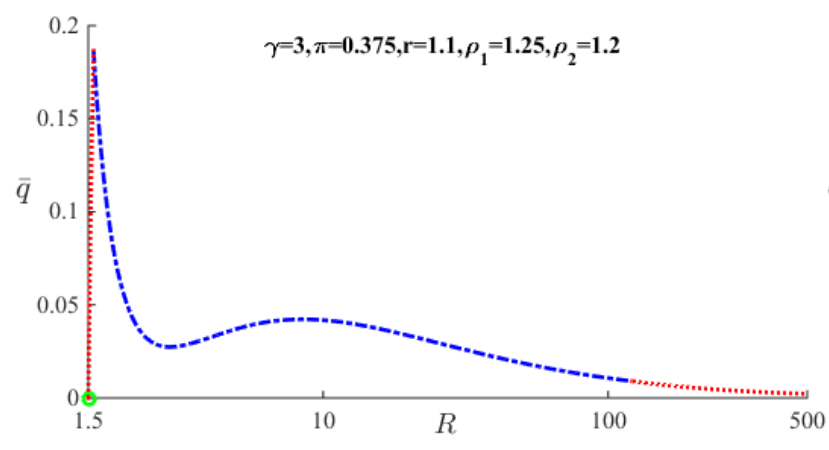

(a) $\bar{q}$ never falls in Case III

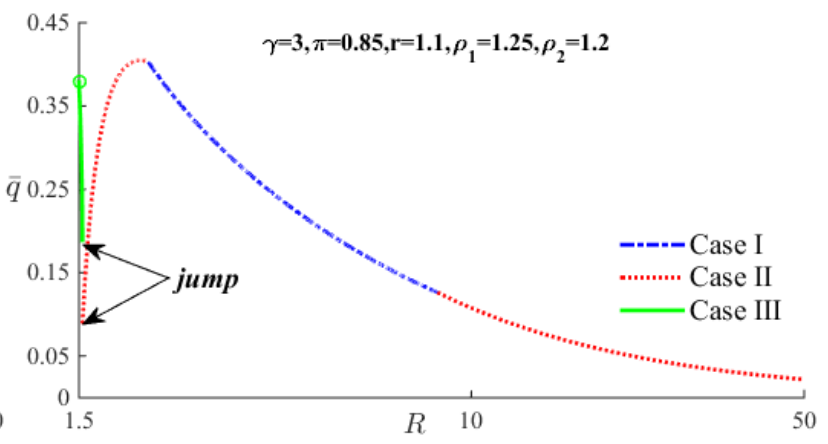

(b) $\bar{q}$ lies in Case III

Figure 7: The impact of $R$ on $\bar{q}$ with $\gamma>2$

It is perhaps instructive to point out how the possibility of a bank run that lies in Case III, with holding excess liquidity and no liquidation, plays an important role in determining the scope for non-monotonicity when $R$ is close to $\rho_{1} \rho_{2}$. It is straightforward to demonstrate that $\bar{q}$ is an increasing function of $R$ because the "state" substitution effect dominates the "period" substitution effect in the neighborhood of $\rho_{1} \rho_{2}$ whenever $\pi$ is sufficient small. Panel (a) of Figure 7 depicts this situation. It is, however, straightforward to show that the net effect formalizes $\bar{q}$ as a decreasing function of $R$ in the neighborhood of $\rho_{1} \rho_{2}$ if the withdrawal demand is sufficiently large, as depicted in panel (b) of Figure 7. Intuitively, holding excess liquidity becomes costly as $R$ rises and, thus, putting more resources in investment will provide a larger return to depositors in period 2. It is clear that an increase in $R$ can promote financial stability in this situation. Also notice that there is a discontinuity between the green and red curve in panel (b), which relates to the existence of "stable region" in the space of $q$ as shown in panel (c) of Figure 5. In other words, an increase in $R$ causes the value of $\bar{q}$ to decrease from $\bar{q}_{\text {Case III }}$ to $\bar{q}_{\text {Case II }}$ discontinuously, which translates into a jump directly from the green curve to the red one. ${ }^{13}$

Unlike the analysis in the neighborhood of $\rho_{1} \rho_{2}$, there does not exist a closed form expression for $c_{1}^{*} / c_{2 \beta}^{*}$ as a general function of $R$. But I can see it is still likely to depend critically on the two competing effects as discussed above. Note further that the $\bar{q}$ might display like a roller-coaster in this environment with $\gamma>2$, which is illustrated by the blue curve in the left panel.

\footnotetext{
${ }^{12} \mathrm{I}$ analyze the impact of asset returns on financial fragility for the cases where $R \rightarrow \rho_{1} \rho_{2}, R \rightarrow \infty, r \rightarrow 0$, $r \rightarrow \rho_{1}$, and $\rho_{2}=1$.

${ }^{13}$ I discuss the existence of this discontinuity in more detail in Appendix C.
} 


\subsection{Implication: liquidation costs and the term premium}

Now, I turn my attention to the impact of changes in the liquidation cost and the term premium on financial fragility in the two-asset model. I define the liquidation cost to be the difference $\rho_{1}-r$ and the term premium to be $R-\rho_{1} \rho_{2}$. There are several ways one might choose to formalize the relationship between $\bar{q}$ and these concepts. One might restrict the changes in term premium with respect to the changes in one of $\rho_{1}, \rho_{2}$ and $R$ holding the other two fixed. Alternatively, for example, one might assume that the changes in term premium stem from any changes in those asset returns. In what follows, I choose the former approach for two reasons: first, it is simpler to work with; and second, the qualitative aspects of my main results provide insight to the latter method.

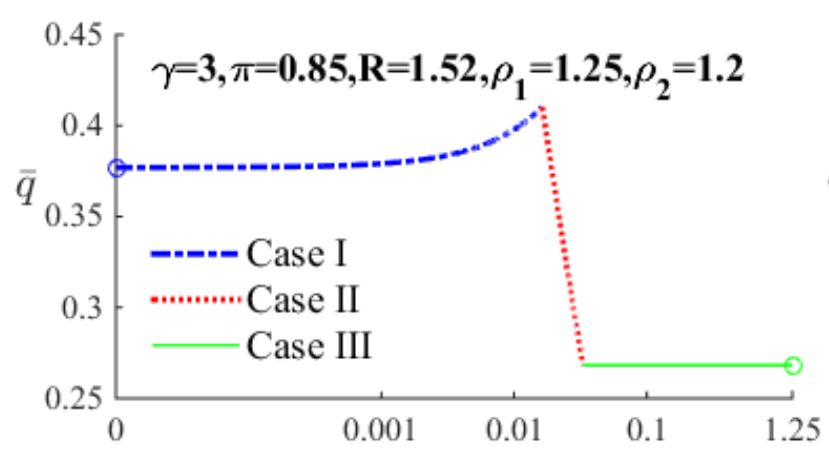

(a) Liquidation cost

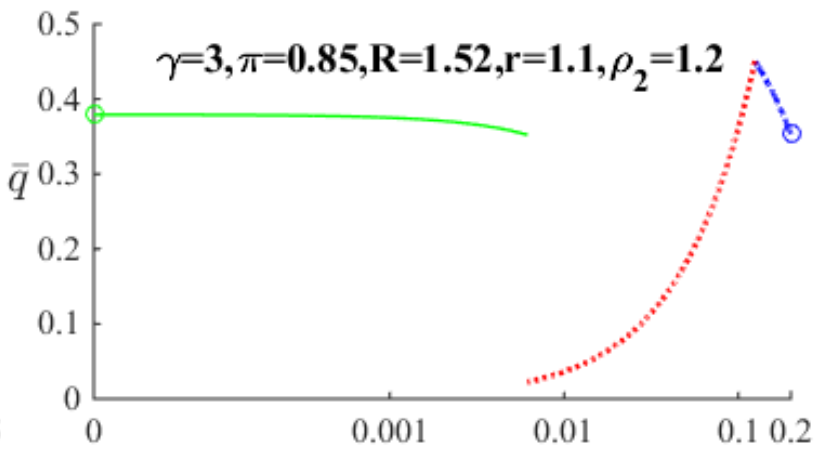

(b) Term premium

Figure 8: The impact of liquidation cost and term premium on $\bar{q}$

Panel (a) of Figure 8 (note the logarithmic scale for the x-axis) illustrates how changes in the liquidation cost influence financial fragility by reducing $r$. When the liquidation cost is sufficiently small, the bank will choose not to hold excess liquidity. In this region, an increase in the liquidation cost has no effect on the bank's portfolio choice and causes a sharp decline in the value of remaining resources if a crisis occurs. This decline, in turn, increases the incentive for patient depositors to withdraw early. As a result, such an increase in the liquidation cost leads to an increase in $\bar{q}$ depicted by a blue curve. As the liquidation cost increases further, however, eventually the bank will choose to hold excess liquidity. In this case, an increase in the liquidation cost leads the bank to raise $c_{2 \beta}$ relative to $c_{1 \beta}$ because providing consumption in period 2 is now relatively less expensive. In addition, depositors who withdraw in period 1 after a run has taken place suffer a smaller "haircut" relative to depositors who were earlier in the order since the bank becomes more conservative. In other words, both effects decrease the ex ante incentive for depositors to run, and hence such an increase in the liquidation cost leads to a decline in $\bar{q}$ depicted by a red curve. When the liquidation cost is sufficiently high, no liquidation becomes the best choice for the bank and, as a result, the ratio $c_{1}^{*} / c_{2 \beta}^{*}$ becomes independent in the liquidation cost. If the economy were 
to satisfy the condition $c_{1}^{*} \geq c_{2 \beta}^{*}$ after the solution set switches into Case III, the resulting property would have $\bar{q}$ holding constant as shown by the green curve.

Panel (b) answers the question of what is the effect of a steeper term structure, with a decline in the short-term rate $\rho_{1}$. Recall that the bank will hold excess liquidity and not liquidate investment if the term premium is close to zero. In this region, an increase in the term premium leads patient depositors to leave their funds in the banking system if a crisis occurs since the "period" substitution effect is always dominant, which reduces the degree of financial fragility in this region. As the term premium increases further, however, eventually the bank will choose to liquidate investment to meet withdrawal demand. In this case, it is straightforward to show financial fragility is determined only by the "state" substitution effect. An increase in the term premium raises the ex ante incentives associated with $c_{1}$ for patient depositors with $i \leq \pi$ to run, which increases financial fragility. Decreasing $\rho_{1}$ further and while fixing other parameter values, I can obtain the opposite result as shown by the blue curve in the left panel.

\section{Conclusion}

In a two-asset version of the Diamond and Dybvig (1983) model with limited commitment, I have analyzed the question of how changes in asset returns influence financial fragility. The relationship turns out to be surprising complex. I first focused on the relationship between financial fragility and the return $R$ on the long-term investment in an environment with no liquidation cost. I found that a higher value of this return may either increase or decrease the degree of financial fragility. Indeed, in some cases, the resulting effect of changes in $R$ on financial fragility is non-monotone. Two competing effects explain this non-monotone pattern. An increase in $R$ leads the bank to raise $c_{2 \beta}$ relative to $c_{1 \beta}$ because providing consumption in period 2 is now relatively less expensive. This fact deceases the ex ante incentive for depositors to run. On the other hand, the bank will choose to hold a more illiquid portfolio since the return on reserves is now relatively lower. This fact implies that depositors who withdraw in period 1 after it becomes clear that a run has taken place suffer a larger "haircut" relative to depositors who were earlier in the order due to the sharp decline in the value of remaining reserves. Thus, the latter effect encourages patient depositors to withdraw early rather than leaving their deposits in the banking system, which tends to make the bank more susceptible to a run. I showed that the non-monotonicity becomes more pronounced when liquidating investment is costly and the bank faces a non-trivial portfolio choice. The pattern can again be understood in terms of two competing effects. Finally, I studied the impact of changes in the liquidation cost and the term premium on financial fragility. Not surprisingly, the pattern discovered here can be non-monotonic as well. 


\section{Appendices}

\section{A The best-response allocation}

In this appendix, I derive the best response of the bank to the strategy profile (1). The expressions derived here are used in the proofs of the propositions given in Appendix B as well as in the general definition of financial fragility presented in Appendix D.

The best response of the bank to profile (1) is the solution to the maximization problem (2):

$$
\max _{\left\{x, c_{1}, c_{1 \beta}, c_{2 \alpha}, c_{2 \beta}\right\}} \pi u\left(c_{1}\right)+(1-q)(1-\pi) u\left(c_{2 \alpha}\right)+q(1-\pi)\left[\hat{\pi}_{\beta} u\left(c_{1 \beta}\right)+\left(1-\hat{\pi}_{\beta}\right) u\left(c_{2 \beta}\right)\right]
$$

subject to the feasibility constraints

$$
\begin{aligned}
\pi c_{1} & \leq \rho_{1}(1-x) \\
(1-\pi) c_{2 \alpha} & =R x+\rho_{2}\left[\rho_{1}(1-x)-\pi c_{1}\right] \\
\rho_{1}(1-x) & \leq \pi c_{1}+(1-\pi) \hat{\pi}_{\beta} c_{1 \beta} \\
(1-\pi)\left(1-\hat{\pi}_{\beta}\right) c_{2 \beta} & =R\left\{x-\frac{1}{r}\left[\pi c_{1}+(1-\pi) \hat{\pi}_{\beta} c_{1 \beta}-\rho_{1}(1-x)\right]\right\}
\end{aligned}
$$

Letting $\mu_{1}, \mu_{2 \alpha}, \mu_{1 \beta}$, and $\mu_{2 \beta}$ demote the multiplier on the constraints, the solution to the problem is characterized by the first-order conditions

$$
\begin{aligned}
u^{\prime}\left(c_{1}\right) & =\mu_{1}+\rho_{2} \mu_{2 \alpha}+\frac{R}{r} \mu_{2 \beta}-\mu_{1 \beta} \\
(1-q) u^{\prime}\left(c_{2 \alpha}\right) & =\mu_{2 \alpha} \\
q u^{\prime}\left(c_{1 \beta}\right) & =\frac{R}{r} \mu_{2 \beta}-\mu_{1 \beta} \\
q u^{\prime}\left(c_{2 \beta}\right) & =\mu_{2 \beta} \\
\mu_{1}+\rho_{2} \mu_{2 \alpha}+\frac{R}{r} \mu_{2 \beta}-\mu_{1 \beta} & =\frac{R}{\rho_{1}}\left(\mu_{2 \alpha}+\mu_{2 \beta}\right) \\
{\left[\pi c_{1}-\rho_{1}(1-x)\right] \mu_{1} } & =0 \\
{\left[\rho_{1}(1-x)-\pi c_{1}-(1-\pi) \hat{\pi}_{\beta} c_{1 \beta}\right] \mu_{1 \beta} } & =0 \\
(1-\pi) c_{2 \alpha} & =R x+\rho_{2}\left[\rho_{1}(1-x)-\pi c_{1}\right] \\
(1-\pi)\left[\hat{\pi}_{\beta} c_{1 \beta}+\left(1-\hat{\pi}_{\beta}\right) \frac{r}{R} c_{2 \beta}\right] & =r x+\rho_{1}(1-x)-\pi c_{1}
\end{aligned}
$$

The solution to the problem will lie in one of three cases, depending on the value of $q$. These cases are: 
Case I: If $0<q<\left\{1+\frac{\rho_{1}-r}{R-\rho_{1} \rho_{2}}\left[\hat{\pi}_{\beta} \frac{R}{r}+\left(1-\hat{\pi}_{\beta}\right)\left(\frac{R}{r}\right)^{\frac{1}{\gamma}}\right]^{\gamma}\right\}^{-1} \equiv q_{l}$, then the solution has $\mu_{1}>0$ and $\mu_{1 \beta}=0$, and is given by:

$$
\begin{aligned}
\frac{c_{1 \beta}^{*}}{c_{2 \beta}^{*}} & =\left(\frac{r}{R}\right)^{\frac{1}{\gamma}}<1 \\
\frac{c_{1}^{*}}{c_{2 \alpha}^{*}} & =\left\{(1-q) \frac{R}{\rho_{1}}+q \frac{R}{\rho_{1}}\left[\hat{\pi}_{\beta}\left(\frac{R}{r}\right)^{1-\frac{1}{\gamma}}+\left(1-\hat{\pi}_{\beta}\right)\right]^{\gamma}\right\}^{-\frac{1}{\gamma}}<1 \\
\frac{c_{1}^{*}}{c_{1 \beta}^{*}} & =\left\{(1-q) \frac{r}{\rho_{1}}\left[\hat{\pi}_{\beta}\left(\frac{R}{r}\right)^{1-\frac{1}{\gamma}}+\left(1-\hat{\pi}_{\beta}\right)\right]^{-\gamma}+q \frac{r}{\rho_{1}}\right\}^{-\frac{1}{\gamma}} \\
\frac{c_{1}^{*}}{c_{2 \beta}^{*}} & =\left\{(1-q) \frac{R}{\rho_{1}}\left[\hat{\pi}_{\beta}\left(\frac{R}{r}\right)^{1-\frac{1}{\gamma}}+\left(1-\hat{\pi}_{\beta}\right)\right]^{-\gamma}+q \frac{R}{\rho_{1}}\right\}^{-\frac{1}{\gamma}}
\end{aligned}
$$

Case II: If $q_{l} \leq q \leq\left\{1+\frac{\rho_{1}-r}{R-\rho_{1} \rho_{2}}\left[\hat{\pi}_{\beta} \rho_{2}+\left(1-\hat{\pi}_{\beta}\right)\left(\frac{R}{r}\right)^{\frac{1}{\gamma}}\right]^{\gamma}\right\}^{-1} \equiv q_{u}$, then the solution has $\mu_{1}=0$ and $\mu_{1 \beta}=0$, and is given by:

$$
\begin{aligned}
\frac{c_{1 \beta}^{*}}{c_{2 \beta}^{*}} & =\left(\frac{r}{R}\right)^{\frac{1}{\gamma}}<1 \\
\frac{c_{1}^{*}}{c_{2 \alpha}^{*}} & =\left[(1-q) \frac{R-r \rho_{2}}{\rho_{1}-r}\right]^{-\frac{1}{\gamma}}<1 \text { as long as } \rho_{2} \geq 1 \\
\frac{c_{1}^{*}}{c_{1 \beta}^{*}} & =\left(q \frac{R-r \rho_{2}}{R-\rho_{1} \rho_{2}}\right)^{-\frac{1}{\gamma}} \\
\frac{c_{1}^{*}}{c_{2 \beta}^{*}} & =\left[q \frac{R\left(R-r \rho_{2}\right)}{r\left(R-\rho_{1} \rho_{2}\right)}\right]^{-\frac{1}{\gamma}}
\end{aligned}
$$

Case III: If $q_{u}<q<1$, then the solution has $\mu_{1}=0$ and $\mu_{1 \beta}>0$, and is given by:

$$
\frac{c_{1 \beta}^{*}}{c_{2 \beta}^{*}}:(1-q)\left(\frac{R}{\rho_{1}}-\rho_{2}\right)\left[\hat{\pi}_{\beta} \rho_{2} \frac{c_{1 \beta}^{*}}{c_{2 \beta}^{*}}+\left(1-\hat{\pi}_{\beta}\right)\right]^{-\gamma}-q\left(\frac{c_{1 \beta}^{*}}{c_{2 \beta}^{*}}\right)^{-\gamma}+q \frac{R}{\rho_{1}}=0
$$

note that $c_{1 \beta}^{*}<c_{2 \beta}^{*}$ always holds as long as $\rho_{2} \geq 1$

$$
\begin{aligned}
& \frac{c_{1}^{*}}{c_{2 \alpha}^{*}}=\left\{(1-q) \frac{R}{\rho_{1}}+q \frac{R}{\rho_{1}}\left[\hat{\pi}_{\beta} \rho_{2} \frac{c_{1 \beta}^{*}}{c_{2 \beta}^{*}}+\left(1-\hat{\pi}_{\beta}\right)\right]^{\gamma}\right\}^{-\frac{1}{\gamma}}<1 \\
& \frac{c_{1}^{*}}{c_{1 \beta}^{*}}=\left\{(1-q) \rho_{2}\left[\hat{\pi}_{\beta} \rho_{2}+\left(1-\hat{\pi}_{\beta}\right) \frac{c_{2 \beta}^{*}}{c_{1 \beta}^{*}}\right]^{-\gamma}+q\right\}^{-\frac{1}{\gamma}} \\
& \frac{c_{1}^{*}}{c_{2 \beta}^{*}}=\left\{(1-q) \frac{R}{\rho_{1}}\left[\hat{\pi}_{\beta} \rho_{2} \frac{c_{1 \beta}^{*}}{c_{2 \beta}^{*}}+\left(1-\hat{\pi}_{\beta}\right)\right]^{-\gamma}+q \frac{R}{\rho_{1}}\right\}^{-\frac{1}{\gamma}}
\end{aligned}
$$




\section{B Proofs of Propositions}

Proposition 1. Suppose $\rho_{1}=r$. If $\pi>\pi_{F}$, then

$$
\bar{q}=\frac{\frac{\rho_{1}}{R}-\left[\pi\left(\frac{R}{\rho_{1}}\right)^{1-\frac{1}{\gamma}}+(1-\pi)\right]^{-\gamma}}{1-\left[\pi\left(\frac{R}{\rho_{1}}\right)^{1-\frac{1}{\gamma}}+(1-\pi)\right]^{-\gamma}} ;
$$

otherwise, $\bar{q}=0$.

Proof. In this economy with no liquidation cost, the solution to the bank's problem is always in Case I and from Appendix A I have

$$
\frac{c_{1}^{*}}{c_{2 \beta}^{*}}=\left\{(1-q) \frac{R}{\rho_{1}}\left[\pi\left(\frac{R}{\rho_{1}}\right)^{1-\frac{1}{\gamma}}+(1-\pi)\right]^{-\gamma}+q \frac{R}{\rho_{1}}\right\}^{-\frac{1}{\gamma}} .
$$

Recalling that $\bar{q}$ is the maximum value of $q$ such that $c_{1}^{*} \geq c_{2 \beta}^{*}$, I can use this expression to calculate

$$
\bar{q}=\left\{\begin{array}{ll}
\frac{\rho_{1}}{R}-\left[\pi\left(\frac{R}{\rho_{1}}\right)^{1-\frac{1}{\gamma}}+(1-\pi)\right]^{-\gamma} & \text { if } \pi>\pi_{F} \\
1-\left[\pi\left(\frac{R}{\rho_{1}}\right)^{1-\frac{1}{\gamma}}+(1-\pi)\right]^{-\gamma} & \text { otherwise }
\end{array}\right\} .
$$

Proposition 2. Suppose $\rho_{1}=r$ and $\pi>\pi_{F}$. If:

- $\pi>\tilde{\pi}$, then $\bar{q}$ is strictly decreasing in $R$;

- $\pi<\tilde{\pi}$, then there exists $\tilde{R}>\rho_{1}$ such that $\bar{q}$ is strictly $\left(\begin{array}{c}\text { increasing } \\ \text { decreasing }\end{array}\right)$ in $R$ as $R\left(\begin{array}{c}< \\ >\end{array}\right) \tilde{R}$.

Proof. The proof of this proposition is divided into four steps as follows.

Step (i): By Proposition 1, the measure of financial fragility $\bar{q}$ is given by

$$
\bar{q}=\left\{\begin{array}{ll}
\frac{\rho_{1}-\left[\pi\left(\frac{R}{R}\right)^{1-\frac{1}{\gamma}}+(1-\pi)\right]^{-\gamma}}{\rho_{1}} & \text { if } \pi>\pi_{F} \\
0 & \text { otherwise }
\end{array}\right\} .
$$

Note that $1-\left[\pi\left(\frac{R}{\rho_{1}}\right)^{1-\frac{1}{\gamma}}+(1-\pi)\right]^{-\gamma}>0$ always holds, while the condition $\pi>\pi_{F}$ would be violated if $1<\gamma \leq 2$. Before deriving the property of $\bar{q}$, I define some useful expressions.

$$
\begin{aligned}
A(R) & \equiv \pi\left(\frac{R}{\rho}\right)^{1-\frac{1}{\gamma}}+(1-\pi)-\left(\frac{R}{\rho_{1}}\right)^{\frac{1}{\gamma}} \\
B(R) & \equiv \frac{\frac{\rho_{1}}{R}-\left[\pi\left(\frac{R}{\rho_{1}}\right)^{1-\frac{1}{\gamma}}+(1-\pi)\right]^{-\gamma}}{1-\left[\pi\left(\frac{R}{\rho_{1}}\right)^{1-\frac{1}{\gamma}}+(1-\pi)\right]^{-\gamma}} \\
C(R) & \equiv(\gamma-1) \pi\left(\frac{R}{\rho_{1}}-1\right)\left(\frac{R}{\rho_{1}}\right)^{1-\frac{1}{\gamma}}\left[\pi\left(\frac{R}{\rho_{1}}\right)^{1-\frac{1}{\gamma}}+(1-\pi)\right]^{-\gamma-1}+\left[\pi\left(\frac{R}{\rho_{1}}\right)^{1-\frac{1}{\gamma}}+(1-\pi)\right]^{-\gamma}-1
\end{aligned}
$$


Differentiating these expressions with respect to $R$, I have

$$
\begin{aligned}
& A^{\prime}(R)=\frac{1}{\gamma}\left(\frac{R}{\rho_{1}}\right)^{\frac{1}{\gamma}-1}\left[(\gamma-1) \pi\left(\frac{R}{\rho_{1}}\right)^{1-\frac{2}{\gamma}}-1\right] \begin{cases}\leq 0 & \text { if } \frac{R}{\rho_{1}} \leq[\pi(\gamma-1)]^{-\frac{\gamma}{\gamma-2}} \\
>0 & \text { otherwise }\end{cases} \\
& B^{\prime}(R)=\frac{C(R)}{\left(\frac{R}{\rho_{1}}\right)^{2}\left\{1-\left[\pi\left(\frac{R}{\rho_{1}}\right)^{1-\frac{1}{\gamma}}+(1-\pi)\right]^{-\gamma}\right\}^{2}} \\
& C^{\prime}(R)=(\gamma-1) \pi\left(\frac{R}{\rho_{1}}-1\right) \frac{\left[\left(2-\frac{1}{\gamma}\right)(1-\pi)-(\gamma-2) \pi\left(\frac{R}{\rho_{1}}\right)^{1-\frac{1}{\gamma}}\right]}{\left(\frac{R}{\rho_{1}}\right)^{\frac{1}{\gamma}}\left[\pi\left(\frac{R}{\rho_{1}}\right)^{1-\frac{1}{\gamma}}+(1-\pi)\right]^{\gamma+2}} \begin{cases}\geq 0 & \text { if } \frac{R}{\rho_{1}} \leq\left[\frac{(1-\pi)\left(2-\frac{1}{\gamma}\right)}{\pi(\gamma-2)}\right] \frac{\gamma}{\gamma-1} \\
<0 & \text { otherwise }\end{cases}
\end{aligned}
$$

It is straightforward to show that

$$
\begin{array}{ll}
\lim _{R \rightarrow \rho_{1}} A(R)=0 & \lim _{R \rightarrow \infty} A(R)=\infty \\
\lim _{R \rightarrow \rho_{1}} B(R)=1-\frac{1}{\pi(\gamma-1)} & \lim _{R \rightarrow \infty} B(R)=0 \\
\lim _{R \rightarrow \rho_{1}} C(R)=0 & \lim _{R \rightarrow \infty} C(R)=-1 \\
\lim _{R \rightarrow \rho_{1}} B^{\prime}(R)=\frac{\left(2-\frac{1}{\gamma}\right)-\left(\gamma-\frac{1}{\gamma}\right) \pi}{2 \pi(\gamma-1)} & \lim _{R \rightarrow \infty} B^{\prime}(R)=0
\end{array}
$$

Step (ii): For $\pi<\frac{1}{\gamma-1}$, using the results from step (i) above, I have

$$
\begin{gathered}
A(R)\left\{\begin{array}{ll}
\leq 0 & \text {, if } \rho_{1}<R \leq \tilde{\tilde{R}} \\
>0 & , \text { if } \tilde{\tilde{R}}<R
\end{array}, \text { where } A(\tilde{\tilde{R}})=0\right. \\
C(R)\left\{\begin{array}{ll}
\geq 0 & \text {, if } \tilde{\tilde{R}}<R \leq \tilde{R} \\
<0 & \text {, if } \tilde{R}<R
\end{array}, \text { where } C(\tilde{R})=0\right.
\end{gathered}
$$

and, hence,

$$
\bar{q} \text { is } \begin{cases}0 & \text {,if } \rho_{1}<R \leq \tilde{\tilde{R}} \\ \text { strictly increasing in } R & , \text { if } \tilde{\tilde{R}}<R \leq \tilde{R} \\ \text { strictly decreasing in } R & , \text { if } \tilde{R}<R\end{cases}
$$

Step (iii): For $\frac{1}{\gamma-1} \leq \pi<\frac{2-\frac{1}{\gamma}}{\gamma-\frac{1}{\gamma}}$, using the results from step (i) above and the fact of $R>\rho_{1}$, it is then straightforward to show that $A(R)>0$, and thus

$$
\bar{q} \text { is strictly } \begin{cases}\text { increasing in } R & \text {, if } \rho_{1}<R \leq \tilde{R} \\ \text { decreasing in } R & \text {, if } \tilde{R}<R\end{cases}
$$

Step (iv): For $\frac{2-\frac{1}{\gamma}}{\gamma-\frac{1}{\gamma}} \leq \pi$, using the results from step (i) above and the fact of $R>\rho_{1}$ again, it then follows that $C(R)<0$, and I have $\bar{q}$ is strictly decreasing in R.

Together, these four steps establish the result. 
Proposition 3. If $\rho_{1}=r$, then $\left(\begin{array}{c}c_{1 \beta}^{*} / c_{2 \beta}^{*} \\ c_{1}^{*} / c_{1 \beta}^{*}\end{array}\right)$ is strictly $\left(\begin{array}{l}\text { decreasing } \\ \text { increasing }\end{array}\right)$ in $R$.

Proof. In this economy with no liquidation cost, the solution to the bank's problem is always in Case I and from Appendix A I have

$$
\begin{aligned}
& \frac{c_{1 \beta}^{*}}{c_{2 \beta}^{*}}=\left(\frac{r}{R}\right)^{\frac{1}{\gamma}}=\left(\frac{\rho_{1}}{R}\right)^{\frac{1}{\gamma}} \\
& \frac{c_{1}^{*}}{c_{1 \beta}^{*}}=\left\{(1-q)\left[\pi\left(\frac{R}{\rho_{1}}\right)^{1-\frac{1}{\gamma}}+(1-\pi)\right]^{-\gamma}+q\right\}^{-\frac{1}{\gamma}}
\end{aligned}
$$

Differentiating these expressions with respect to $R$ gives the desired result.

Proposition 4. Given $R, r, \rho_{1}, \rho_{2}, \gamma$, and $\pi$,

- if $f(\cdot) \leq 0$, then the economy is stable for all $q$ and, therefore, $\bar{q}=0$;

- if $f(\cdot)>0$ and $g(\cdot) \leq 0$, then

$$
\bar{q}=\frac{\frac{\rho_{1}}{R}-\left[\pi\left(\frac{R}{r}\right)^{1-\frac{1}{\gamma}}+(1-\pi)\right]^{-\gamma}}{1-\left[\pi\left(\frac{R}{r}\right)^{1-\frac{1}{\gamma}}+(1-\pi)\right]^{-\gamma}} \equiv \bar{q}_{\text {Case I }}
$$

- if $g(\cdot)>0$ and $\left\{q \mid h(q)=0 ; q \in\left(q_{u}, 1\right)\right\}=\emptyset$, then

$$
\bar{q}=\frac{\left(R-\rho_{1} \rho_{2}\right) r}{\left(R-\rho_{2} r\right) R} \equiv \bar{q}_{\text {Case II }}
$$

- if $g(\cdot)>0$ and $\left\{q \mid h(q)=0 ; q \in\left(q_{u}, 1\right)\right\}=\mathcal{Q}\left\{q_{1}, q_{2}, \ldots, q_{n}\right\}$, then

$$
\bar{q}=q_{m} \equiv \bar{q}_{\text {Case III }} \text {, where } q_{m} \text { is the biggest element of } \mathcal{Q} \text {. }
$$

Proof. The measure of financial fragility $\bar{q}$ will lie in Cases I, II, and III depending on parameter values. Define

$$
\begin{aligned}
f(\cdot) & =\left[\pi\left(\frac{R}{r}\right)^{1-\frac{1}{\gamma}}+(1-\pi)\right]^{\gamma}-\frac{R}{\rho_{1}} \\
g(\cdot) & =\left[\pi\left(\frac{R}{r}\right)^{1-\frac{1}{\gamma}}+(1-\pi)\right]^{\gamma}-\frac{R^{2}-\left(\rho_{2}+1\right) r R+\rho_{1} \rho_{2} r}{R\left(\rho_{1}-r\right)} \\
h(q) & =\pi \rho_{2}\left(\rho_{2}+\frac{1-\frac{\rho_{1} \rho_{2}}{R}}{q}\right)^{-\frac{1}{\gamma}}+(1-\pi)-\left[\frac{(1-q) \frac{R}{\rho_{1}}}{1-q \frac{R}{\rho_{1}}}\right]^{\frac{1}{\gamma}} .
\end{aligned}
$$

There are four scenarios needed to be considered. 
Scenario (i): If $f(\cdot) \leq 0$, using Appendix A, then I see that $c_{1}^{*}<c_{2 \beta}^{*}$ holds in Cases I, II, and III under this condition. Hence, I have $\bar{q}=0$.

Scenario (ii): If $f(\cdot)>0$ and $g(\cdot) \leq 0$, using Appendix A, then I see that $c_{1}^{*}<c_{2 \beta}^{*}$ holds in Cases II, and III under this condition. It is straightforward to show that $\frac{c_{1}^{*}}{c_{2 \beta}^{*}}$ is strictly decreasing in $q$ when the solution lies in Case I. In this case, $c_{1}^{*} \geq c_{2 \beta}^{*}$ if and only if $q \leq \frac{\frac{\rho_{1}}{R}-\left[\pi\left(\frac{R}{r}\right)^{1-\frac{1}{\gamma}}+(1-\pi)\right]^{-\gamma}}{1-\left[\pi\left(\frac{R}{r}\right)^{1-\frac{1}{\gamma}}+(1-\pi)\right]^{-\gamma}}$. Hence, I have $\bar{q}=\frac{\frac{\rho_{1}}{R}-\left[\pi\left(\frac{R}{r}\right)^{1-\frac{1}{\gamma}}+(1-\pi)\right]^{-\gamma}}{1-\left[\pi\left(\frac{R}{r}\right)^{1-\frac{1}{\gamma}}+(1-\pi)\right]^{-\gamma}}$.

Scenario (iii): If $g(\cdot)>0$ and $\left\{q \mid h(q)=0 ; q \in\left(q_{u}, 1\right)\right\}=\emptyset$, using Appendix A, then I see that $c_{1}^{*}>c_{2 \beta}^{*}$ holds in Case I and $c_{1}^{*}<c_{2 \beta}^{*}$ holds in Case III under this condition. It is straightforward to show that $\frac{c_{1}^{*}}{c_{2 \beta}^{*}}$ is strictly decreasing in $q$ when the solution lies in Case II. In this case, $c_{1}^{*} \geq c_{2 \beta}^{*}$ if and only if $q \leq \frac{r\left(R-\rho_{1} \rho_{2}\right)}{R\left(R-r \rho_{2}\right)}$. Hence, I have $\bar{q}=\frac{r\left(R-\rho_{1} \rho_{2}\right)}{R\left(R-r \rho_{2}\right)}$.

Scenario (iv): If $g(\cdot)>0$ and $\left\{q \mid h(q)=0 ; q \in\left(q_{u}, 1\right)\right\} \neq \emptyset$, using Appendix A and Definition 2, then I see that $\bar{q}$ lies in Case III, as shown in panels (c) and (d) of Figure 5. It is straightforward to show that there exists a vector $\mathcal{Q}$ such that $\frac{c_{1}^{*}}{c_{2 \beta}^{*}}(\mathcal{Q})=1$. Let $q_{m}$ be the largest element of $\mathcal{Q}$. Then, I have $\bar{q}=q_{m}$.

Proposition 5. Assume $f(\cdot)>0$ holds. For all $\gamma \in(1,2]$,

$\bar{q}$ is strictly $\left\{\begin{array}{l}\left(\begin{array}{c}\text { increasing } \\ \text { decreasing }\end{array}\right) \text { in } R \text { with } R\left(\begin{array}{c}< \\ >\end{array}\right) \min \left\{R^{*}, \underline{R}\right\}, \text { if } \pi<\left(\frac{r}{\rho_{1}-r}\right)^{\frac{1}{\gamma}} \cdot \mathbb{1}\{\gamma=2\} \\ \left(\begin{array}{c}\text { increasing } \\ \text { decreasing }\end{array}\right) \text { in } R \text { with } R\left(\begin{array}{c}< \\ >\end{array}\right) \underline{R}, \quad \text { if } \pi>\left(\frac{r}{\rho_{1}-r}\right)^{\frac{1}{\gamma}} \cdot \mathbb{1}\{\gamma=2\}\end{array}\right\}$,

$\mathbb{1}\{\gamma=2\}$ is an indicator function, where $\mathbb{1}\{\gamma=2\}=1$ if $\gamma=2$ and 0 otherwise.

Proof. Consider any economy with $1<\gamma \leq 2$. Notice that $\frac{c_{1}^{*}}{c_{2 \beta}^{*}}$ is increasing in $\rho_{2}$ in Case III. Suppose that $\rho_{2}=\frac{R}{\rho_{1}}$, I then have $\frac{c_{1}^{*}}{c_{2 \beta}^{*}}<1$ in Case III since the condition $\pi>\pi_{F}$ is violated. In other words, I have the following results:

- if $f(\cdot) \leq 0$, then the economy is stable and, therefore, $\bar{q}=0$;

- if $f(\cdot)>0$ and $g(\cdot) \leq 0$, then

$$
\bar{q}=\frac{\frac{\rho_{1}}{R}-\left[\pi\left(\frac{R}{r}\right)^{1-\frac{1}{\gamma}}+(1-\pi)\right]^{-\gamma}}{1-\left[\pi\left(\frac{R}{r}\right)^{1-\frac{1}{\gamma}}+(1-\pi)\right]^{-\gamma}} \equiv \bar{q}_{\text {Case I }} ;
$$

- if $g(\cdot)>0$, then

$$
\bar{q}=\frac{\left(R-\rho_{1} \rho_{2}\right) r}{\left(R-\rho_{2} r\right) R} \equiv \bar{q}_{\text {Case II }}
$$


Taking the derivatives of $\bar{q}_{\text {Case I }}$ and $\bar{q}_{\text {Case II }}$ with respective to $R$, yields the following results.

\begin{tabular}{c|c}
\hline & $R$ \\
\hline $\bar{q}_{\text {Case I }}$ & $\downarrow$ \\
\hline $\bar{q}_{\text {Case II }}$ & $\left\{\begin{array}{l}\uparrow \\
\downarrow\end{array}\right\}$ if $R\left\{\begin{array}{l}< \\
>\end{array}\right\} \underline{R}$ \\
\hline
\end{tabular}

where $\uparrow(\downarrow)$ represents an increasing (decreasing) function;

Table 2: The impact of the return $R$ on $\bar{q}$ with $1<\gamma \leq 2$

Next, I can identify $\bar{q}$ by using the sign of $f(\cdot)$ and $g(\cdot)$.

- If $\pi<\frac{\rho_{2}^{\frac{1}{\gamma}}-1}{\left(\frac{\rho_{1} \rho_{2}}{r}\right)^{1-\frac{1}{\gamma}}}$, I see that $f(\cdot) \leq 0$. Thus, I have $\bar{q}=0$.

- If $\frac{\rho_{2}^{\frac{1}{\gamma}}-1}{\left(\frac{\rho_{1} \rho_{2}}{r}\right)^{1-\frac{1}{\gamma}}} \leq \pi<\left(\frac{r}{\rho_{1}}\right)^{\frac{1}{\gamma} \cdot 1\{\gamma=2\}}$, I see that

$$
\left\{\begin{array}{c}
f(\cdot) \leq 0 \\
f(\cdot)>0 \text { and } g(\cdot) \leq 0 \\
g(\cdot)>0
\end{array}\right\} \text { if } R \in\left\{\begin{array}{c}
\left(R^{* *}, \infty\right) \\
\left(R^{*}, R^{* *}\right) \\
\left(\rho_{1} \rho_{2}, R^{*}\right)
\end{array}\right\} \text {, i.e. } \bar{q}=\left\{\begin{array}{c}
0 \\
\bar{q}_{\text {Case I I }} \\
\bar{q}_{\text {Case II }}
\end{array}\right\} \text { if } R \in\left\{\begin{array}{l}
\left(R^{* *}, \infty\right) \\
\left(R^{*}, R^{* *}\right) \\
\left(\rho_{1} \rho_{2}, R^{*}\right)
\end{array}\right\}
$$

where $f\left(R^{* *}\right)=0$ and $g\left(R^{*}\right)=0$.

- If $\left(\frac{r}{\rho_{1}}\right)^{\frac{1}{\gamma}} \cdot \mathbb{1}\{\gamma \equiv 2\} \leq \pi<\left(\frac{r}{\rho_{1}-r}\right)^{\frac{1}{\gamma} \cdot 1\{\gamma \equiv 2\}}$, I see that

$$
\left\{\begin{array}{c}
f(\cdot)>0 \text { and } g(\cdot) \leq 0 \\
g(\cdot)>0
\end{array}\right\} \text { if } R\left\{\begin{array}{l}
> \\
<
\end{array}\right\} R^{*} \text {, i.e. } \bar{q}=\left\{\begin{array}{c}
\bar{q}_{\text {Case I }} \\
\bar{q}_{\text {Case II }}
\end{array}\right\} \text { if } R\left\{\begin{array}{l}
> \\
<
\end{array}\right\} R^{*}
$$

- If $\left(\frac{r}{\rho_{1}-r}\right)^{\frac{1}{\gamma} \cdot 1\{\gamma \equiv 2\}} \leq \pi$, I see that $g(\cdot)>0$. Thus, I have $\bar{q}=\bar{q}_{\text {Case II }}$.

Combined with the results of Table 2, I then have this proposition, as desired.

Proposition 6. Assume $f(\cdot)>0$ holds. For all $\gamma \in(1,2]$,

- $\bar{q}$ is strictly $\left\{\begin{array}{l}\text { increasing } \\ \text { decreasing }\end{array}\right\}$ in $r$ with $r\left\{\begin{array}{c}< \\ >\end{array}\right\} r^{*}$

- $\bar{q}$ is strictly $\left\{\begin{array}{l}\text { increasing } \\ \text { decreasing }\end{array}\right\}$ in $\rho_{1}$ with $\rho_{1}\left\{\begin{array}{l}< \\ >\end{array}\right\} \rho_{1}^{*}$

- $\bar{q}$ is $\left\{\begin{array}{lll}\left(\begin{array}{c}\text { constant } \\ \text { decreasing }\end{array}\right) & \text { in } \rho_{2} \quad \text { with } \rho_{2}\left(\begin{array}{c}< \\ >\end{array}\right) \rho_{2}^{*}, & \text { if } \pi<\pi^{*} \\ \text { decreasing } & \text { in } \rho_{2}, & \text { if } \pi>\pi^{*}\end{array}\right\}$ 
Proof. Consider any economy with $1<\gamma \leq 2$. Notice that $\frac{c_{1}^{*}}{c_{2 \beta}^{*}}$ is increasing in $\rho_{2}$ in Case III. Suppose that $\rho_{2}=\frac{R}{\rho_{1}}$, I then have $\frac{c_{1}^{*}}{c_{2 \beta}^{*}}<1$ in Case III since the condition $\pi>\pi_{F}$ is violated. In other words, I have the following results:

- if $f(\cdot) \leq 0$, then the economy is stable and, therefore, $\bar{q}=0$;

- if $f(\cdot)>0$ and $g(\cdot) \leq 0$, then

$$
\bar{q}=\frac{\frac{\rho_{1}}{R}-\left[\pi\left(\frac{R}{r}\right)^{1-\frac{1}{\gamma}}+(1-\pi)\right]^{-\gamma}}{1-\left[\pi\left(\frac{R}{r}\right)^{1-\frac{1}{\gamma}}+(1-\pi)\right]^{-\gamma}} \equiv \bar{q}_{\text {Case I }} ;
$$

- if $g(\cdot)>0$, then

$$
\bar{q}=\frac{\left(R-\rho_{1} \rho_{2}\right) r}{\left(R-\rho_{2} r\right) R} \equiv \bar{q}_{\text {Case II }}
$$

Taking the derivatives of $\bar{q}_{\text {Case I }}$ and $\bar{q}_{\text {Case II }}$ with respective to $r, \rho_{1}$, and $\rho_{2}$, yields the following results.

\begin{tabular}{c|c|c|c}
\hline & $r$ & $\rho_{1}$ & $\rho_{2}$ \\
\hline $\bar{q}_{\text {Case I }}$ & $\downarrow$ & $\uparrow$ & $\varnothing$ \\
\hline $\bar{q}_{\text {Case II }}$ & $\uparrow$ & $\downarrow$ & $\downarrow$ \\
\hline
\end{tabular}

where $\uparrow(\downarrow)$ represents an increasing (decreasing) function; $\varnothing$ represents no effect;

Table 3: The impact of asset returns on $\bar{q}$ with $1<\gamma \leq 2$

Next, I can identify $\bar{q}$ by using the sign of $f(\cdot)$ and $g(\cdot)$ with respective to $r, \rho_{1}$, and $\rho_{2}$.

- $r$ :

$$
\left\{\begin{array}{c}
f(\cdot) \leq 0 \\
f(\cdot)>0 \text { and } g(\cdot) \leq 0 \\
g(\cdot)>0
\end{array}\right\} \text { if } r \in\left\{\begin{array}{c}
\left(r^{* *}, \rho_{1}\right) \\
\left(r^{*}, r^{* *}\right) \\
\left(0, r^{*}\right)
\end{array}\right\} \text {, i.e. } \bar{q}=\left\{\begin{array}{c}
0 \\
\bar{q}_{\text {Case I }} \\
\bar{q}_{\text {Case II }}
\end{array}\right\} \text { if } r \in\left\{\begin{array}{c}
\left(r^{* *}, \rho_{1}\right) \\
\left(r^{*}, r^{* *}\right) \\
\left(0, r^{*}\right)
\end{array}\right\}
$$

where $f\left(r^{* *}\right)=0$ and $g\left(r^{*}\right)=0$.

- $\rho_{1}$ :

- If $\pi<\frac{\rho_{2}^{\frac{1}{\gamma}}-1}{\left(\frac{R}{r}\right)^{1-\frac{1}{\gamma}}-1}$, I see that $f(\cdot) \leq 0$. Thus, I have $\bar{q}=0$.

- If $\frac{\rho_{2}^{\frac{1}{\gamma}}-1}{\left(\frac{R}{r}\right)^{1-\frac{1}{\gamma}}-1} \leq \pi$, I see that

$$
\left\{\begin{array}{c}
f(\cdot) \leq 0 \\
f(\cdot)>\text { and } g(\cdot) \leq 0 \\
g(\cdot)>0
\end{array}\right\} \text { if } \rho_{1} \in\left\{\begin{array}{c}
\left(r, \rho_{1}^{* *}\right) \\
\left(\rho_{1}^{* *}, \rho_{1}^{*}\right) \\
\left(\rho_{1}^{*}, \frac{R}{\rho_{2}}\right)
\end{array}\right\} \text {, i.e. } \bar{q}=\left\{\begin{array}{c}
0 \\
\bar{q}_{\text {Case I }} \\
\bar{q}_{\text {Case II }}
\end{array}\right\} \text { if } \rho_{1} \in\left\{\begin{array}{c}
\left(r, \rho_{1}^{* *}\right) \\
\left(\rho_{1}^{* *}, \rho_{1}^{*}\right) \\
\left(\rho_{1}^{*}, \frac{R}{\rho_{2}}\right)
\end{array}\right\}
$$

where $f\left(\rho_{1}^{* *}\right)=0$ and $g\left(\rho_{1}^{*}\right)=0$. 
- $\rho_{2}$ :

- If $\pi<\frac{\left(\frac{R}{\rho_{1}}\right)^{\frac{1}{\gamma}}-1}{\left(\frac{R}{r}\right)^{1-\frac{1}{\gamma}}-1}$, I see that $f(\cdot) \leq 0$. Thus, I have $\bar{q}=0$.

- If $\frac{\left(\frac{R}{\rho_{1}}\right)^{\frac{1}{\gamma}}-1}{\left(\frac{R}{r}\right)^{1-\frac{1}{\gamma}}-1} \leq \pi<\frac{\left[\frac{R^{2}-2 r R+\rho_{1} r}{R\left(\rho_{1}-r\right)}\right]^{\frac{1}{\gamma}}-1}{\left(\frac{R}{r}\right)^{1-\frac{1}{\gamma}}-1}$, I see that

$$
\left\{\begin{array}{c}
f(\cdot)>0 \text { and } g(\cdot) \leq 0 \\
g(\cdot)>0
\end{array}\right\} \text { if } \rho_{2}\left\{\begin{array}{c}
< \\
>
\end{array}\right\} \rho_{2}^{*} \text {, i.e. } \bar{q}=\left\{\begin{array}{c}
\bar{q}_{\text {Case I }} \\
\bar{q}_{\text {Case II }}
\end{array}\right\} \text { if } \rho_{2}\left\{\begin{array}{l}
< \\
>
\end{array}\right\} \rho_{2}^{*}
$$

where $g\left(\rho_{2}^{*}\right)=0$.

- If $\frac{\left[\frac{R^{2}-2 r R+\rho_{1} r}{R\left(\rho_{1}-r\right)}\right]^{\frac{1}{\gamma}}-1}{\left(\frac{R}{r}\right)^{1-\frac{1}{\gamma}}-1} \leq \pi$, I see that $g(\cdot)>0$. Thus, I have $\bar{q}=\bar{q}_{\text {Case II }}$.

Combined with the results of Table 3, I then have this proposition, as desired. 


\section{C "Stable region"}

In this appendix, I illustrate how, in some cases, the economy can be stable for an interval of values of $q$ less than $\bar{q}$. Lemma 1 shows that the bank's best response lies in Case I, Case II, and Case III respectively as $q$ increases. It is straightforward to show that $\frac{c_{1}^{*}}{c_{2 \beta}^{*}}$ is decreasing in $q$ because of costly liquidation whenever the bank's best response lies in Case I or Case II. Things are different when $q \geq q_{u}$. In this situation, the bank becomes more cautious and holds sufficient short assets in portfolio so that it avoids the loss of liquidating investment. Figure 9 illustrates that an increase in the probability of a crisis can either increase or decrease the ratio $\frac{c_{1}^{*}}{c_{2 \beta}^{*}}$ under Case III, depending on parameter values. In addition, the value of $\bar{q}$ decreases discontinuously.

The left panel of Figure 9 shows that there is a "stable region" (i.e. the economy is stable for an interval of values of $q$ less that $\bar{q}$ ) that lies in between 0 and $\bar{q}$. Moreover, increasing $R$ slightly, (for example, increasing $R$ from 1.2 to 1.3 ) the economies in the whole region of Case III becomes stable as depicted in the right panel, which captures the fact that an increase $R$ causes the value of $\bar{q}$ to decrease from $\bar{q}_{\text {Case III }}$ to $\bar{q}_{\text {Case II }}$ discontinuously. (See the "jump" illustrated by panel (b) of Figure 7.)

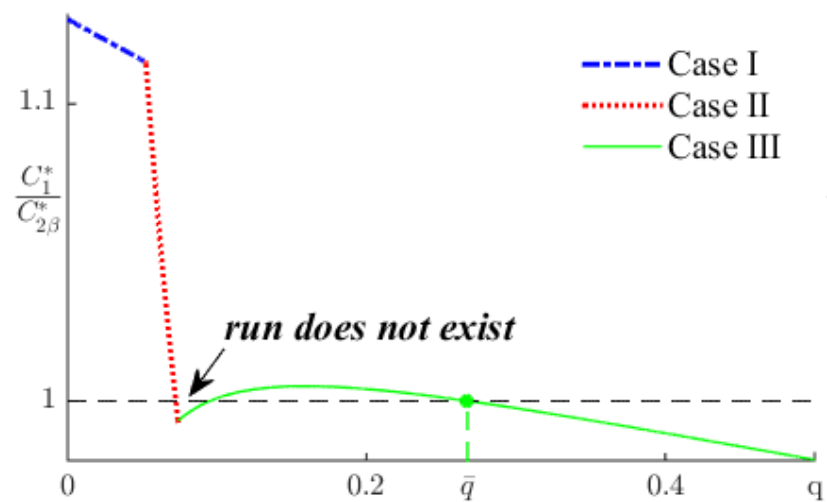

(a) $R=1.2$

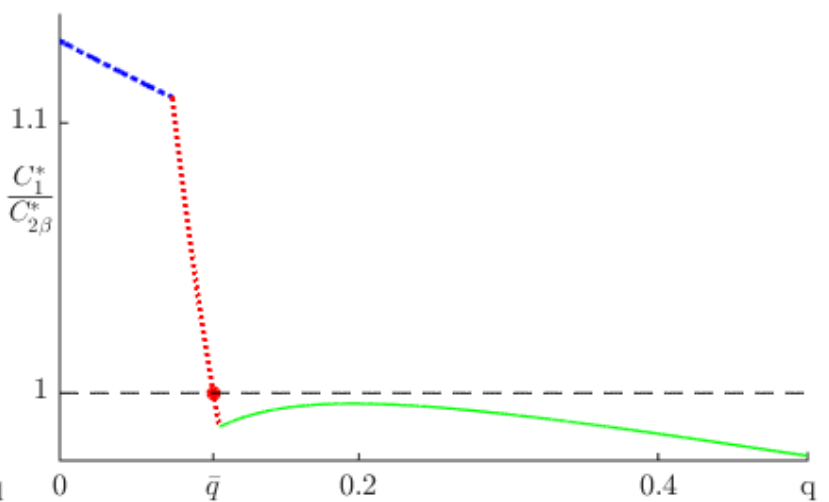

(b) $R=1.3$

Figure 9: $\frac{c_{1}^{*}}{c_{2 \beta}^{*}}$ in the economy $\left(\gamma=3, \pi=0.85, r=1.1, \rho_{1}=1.25, \rho_{2}=1.2\right)$ with $R$ varied 


\section{The general case $\gamma>2$}

Given the complexity of the function $h(q)$, I present some analytical results for the comparative statics in the complex case where $\gamma>2$ in this appendix.

Following the same underlying logic as in the proof of Propositions 5 and 6 , I can identify $\bar{q}$ by using the sign of $f(\cdot)$ and $g(\cdot)$, and the nature of $h(q)$ with respective to $R, r, \rho_{1}$, and $\rho_{2}$. There exist $\epsilon>0$ and $\mathcal{R}>\rho_{1} \rho_{2}$ such that:

- $R$ :

- If $\pi<\frac{\rho_{2}^{\frac{1}{\gamma}}-1}{\left(\frac{\rho_{1} \rho_{2}}{r}\right)^{1-\frac{1}{\gamma}}-1}$, I see that

$$
\bar{q}=\left\{\begin{array}{c}
0 \\
\bar{q}_{\text {Case I }} \\
\bar{q}_{\text {Case II }}
\end{array}\right\} \text { if } R \in\left\{\begin{array}{c}
\left(\rho_{1} \rho_{2}, R^{* *}\right) \\
\left(R^{* *}, R^{* *}+\epsilon\right) \\
(\mathcal{R}, \infty)
\end{array}\right\}
$$

- If $\frac{\rho_{2}^{\frac{1}{\gamma}}-1}{\left(\frac{\rho_{1} \rho_{2}}{r}\right)^{1-\frac{1}{\gamma}}-1} \leq \pi<\frac{\rho_{2}^{\frac{1}{\gamma}}-1}{\rho_{2}-\frac{1}{\gamma}-1}$, I see that

$$
\bar{q}=\bar{q}_{\text {Case II }} \text {, if } R \in\left(\rho_{1} \rho_{2}, \rho_{1} \rho_{2}+\epsilon\right) \text { or }(\mathcal{R}, \infty)
$$

- If $\frac{\rho_{2}^{\frac{1}{\gamma}}-1}{\rho_{2}-\frac{1}{\gamma}-1} \leq \pi$, I see that

$$
\bar{q}=\left\{\begin{array}{c}
q_{m} \\
\bar{q}_{\text {Case II }}
\end{array}\right\} \text { if } R \in\left\{\begin{array}{c}
\left(\rho_{1} \rho_{2}, \rho_{1} \rho_{2}+\epsilon\right) \\
(\mathcal{R}, \infty)
\end{array}\right\}
$$

- $r$ :

- If $\pi<\frac{\left(\frac{R}{\rho_{1}}\right)^{\frac{1}{\gamma}}-1}{\left(\frac{R}{\rho_{1}}\right)^{1-\frac{1}{\gamma}}-1}$, I see that

$$
\bar{q}=\left\{\begin{array}{c}
0 \\
\bar{q}_{\text {Case I }} \\
\bar{q}_{\text {Case II }}
\end{array}\right\} \text { if } r \in\left\{\begin{array}{c}
\left(r^{* *}, \rho_{1}\right) \\
\left(r^{*}, r^{* *}\right) \\
\left(0, r^{*}\right)
\end{array}\right\}
$$

- If $\frac{\left(\frac{R}{\rho_{1}}\right)^{\frac{1}{\gamma}}-1}{\left(\frac{R}{\rho_{1}}\right)^{1-\frac{1}{\gamma}}-1} \leq \pi$ and $\{q \mid h(q)=0 ; q \in(0,1)\}=\emptyset$, I see that

$$
\bar{q}=\left\{\begin{array}{c}
\bar{q}_{\text {Case I }} \\
\bar{q}_{\text {Case II }}
\end{array}\right\} \text { if } r\left\{\begin{array}{l}
> \\
<
\end{array}\right\} r^{*}
$$

- If $\frac{\left(\frac{R}{\rho_{1}}\right)^{\frac{1}{\gamma}}-1}{\left(\frac{R}{\rho_{1}}\right)^{1-\frac{1}{\gamma}}-1} \leq \pi$ and $\{q \mid h(q)=0 ; q \in(0,1)\} \neq \emptyset$, I see that

$$
\bar{q}=\left\{\begin{array}{c}
\bar{q}_{\text {Case I }} \\
\bar{q}_{\text {Case II }} \\
q_{m}
\end{array}\right\} \text { if } r \in\left\{\begin{array}{c}
\left(r^{*}, \rho_{1}\right) \\
\left(r^{*}-\epsilon, r^{*}\right) \\
(0, \epsilon)
\end{array}\right\}
$$


- $\rho_{1}$ :

- If $\pi<\frac{\rho_{2}^{\frac{1}{\gamma}}-1}{\left(\frac{R}{r}\right)^{1-\frac{1}{\gamma}}-1}$, I see that $f(\cdot) \leq 0$. Thus, I have $\bar{q}=0$.

- If $\frac{\rho_{2}^{\frac{1}{\gamma}}-1}{\left(\frac{R}{r}\right)^{1-\frac{1}{\gamma}}-1} \leq \pi<\frac{\left(\frac{R}{r}\right)^{\frac{1}{\gamma}}-1}{\left(\frac{R}{r}\right)^{1-\frac{1}{\gamma}}-1}$, I see that

$$
\bar{q}=\left\{\begin{array}{c}
0 \\
\bar{q}_{\text {Case I }} \\
\bar{q}_{\text {Case II }}
\end{array}\right\} \text { if } \rho_{1} \in\left\{\begin{array}{c}
\left(r, \rho_{1}^{* *}\right) \\
\left(\rho_{1}^{* *}, \rho_{1}^{*}\right) \\
\left(\rho_{1}^{*}, \frac{R}{\rho_{2}}\right)
\end{array}\right\}
$$

- If $\frac{\left(\frac{R}{r}\right)^{\frac{1}{\gamma}}-1}{\left(\frac{R}{r}\right)^{1-\frac{1}{\gamma}}-1} \leq \pi<\frac{\rho_{2}^{\frac{1}{\gamma}}-1}{\rho_{2}^{1-\frac{1}{\gamma}}-1}$, I see that

$$
\bar{q}=\left\{\begin{array}{c}
\bar{q}_{\text {Case I }} \\
\bar{q}_{\text {Case II }}
\end{array}\right\} \text { if } \rho_{1}\left\{\begin{array}{l}
< \\
>
\end{array}\right\} \rho_{1}^{*}
$$

- If $\frac{\rho_{2}^{\frac{1}{\gamma}}-1}{\rho_{2}^{1-\frac{1}{\gamma}}-1} \leq \pi$, I see that

$$
\bar{q}=\left\{\begin{array}{c}
\bar{q}_{\text {Case I }} \\
\bar{q}_{\text {Case II }} \\
q_{m}
\end{array}\right\} \text { if } \rho_{1} \in\left\{\begin{array}{c}
\left(r, \rho_{1}^{*}\right) \\
\left(\rho_{1}^{*}, \rho_{1}^{*}+\epsilon\right) \\
\left(\frac{R}{\rho_{2}}-\epsilon, \frac{R}{\rho_{2}}\right)
\end{array}\right\}
$$

- $\rho_{2}$ :

- If $\pi<\frac{\left(\frac{R}{\rho_{1}}\right)^{\frac{1}{\gamma}}-1}{\left(\frac{R}{r}\right)^{1-\frac{1}{\gamma}}-1}$, I see that $f(\cdot) \leq 0$. Thus, I have $\bar{q}=0$.

- If $\frac{\left(\frac{R}{\rho_{1}}\right)^{\frac{1}{\gamma}}-1}{\left(\frac{R}{r}\right)^{1-\frac{1}{\gamma}}-1} \leq \pi<\frac{\left[\frac{R^{2}-2 r R+\rho_{1} r}{R\left(\rho_{1}-r\right)}\right]^{\frac{1}{\gamma}}-1}{\left(\frac{R}{r}\right)^{1-\frac{1}{\gamma}}-1}$, I see that

$$
\bar{q}=\bar{q}_{\text {Case I }} \text { if } \rho_{2} \in\left[1, \rho_{2}^{*}\right), \text { where } g\left(\rho_{2}^{*}\right)=0
$$

- If $\frac{\left[\frac{R^{2}-2 r R+\rho_{1} r}{R\left(\rho_{1}-r\right)}\right]^{\frac{1}{\gamma}}-1}{\left(\frac{R}{r}\right)^{1-\frac{1}{\gamma}}-1} \leq \pi$, I see that

$$
\bar{q}=\bar{q}_{\text {Case II }} \text { if } \rho_{2} \in[1,1+\epsilon)
$$

- If $\frac{\left(\frac{R}{\rho_{1}}\right)^{\frac{1}{\gamma}}-1}{\left(\frac{R}{r}\right)^{1-\frac{1}{\gamma}}-1} \leq \pi<\frac{\left(\frac{R}{\rho_{1}}\right)^{\frac{1}{\gamma}}-1}{\left(\frac{R}{\rho_{1}}\right)^{1-\frac{1}{\gamma}}-1}$, I see that

$$
\bar{q}=\bar{q}_{\text {Case II }} \text { if } \rho_{2} \in\left(\frac{R}{\rho_{1}}-\epsilon, \frac{R}{\rho_{1}}\right)
$$

- If $\frac{\left(\frac{R}{\rho_{1}}\right)^{\frac{1}{\gamma}}-1}{\left(\frac{R}{\rho_{1}}\right)^{1-\frac{1}{\gamma}}-1} \leq \pi$, I see that

$$
\bar{q}=q_{m} \text { if } \rho_{2} \in\left(\frac{R}{\rho_{1}}-\epsilon, \frac{R}{\rho_{1}}\right)
$$


Taking the derivatives of $\bar{q}_{\text {Case I }}$ and $\bar{q}_{\text {Case II }}$ with respective to $R, r, \rho_{1}, \rho_{2}$, yields the following results.

\begin{tabular}{c|c|c|c|c}
\hline & $R$ & $r$ & $\rho_{1}$ & $\rho_{2}$ \\
\hline $\bar{q}_{\text {Case I }}$ & $\uparrow$ if $R \in\left(R^{* *}, R^{* *}+\epsilon\right)$ & $\downarrow$ & $\uparrow$ & $\varnothing$ \\
\hline $\bar{q}_{\text {Case II }}$ & $\left\{\begin{array}{l}\uparrow \\
\downarrow\end{array}\right\}$ if $R\left\{\begin{array}{l}< \\
>\end{array}\right\}$ & $\uparrow$ & $\downarrow$ & $\downarrow$ \\
\hline$q_{m}$ & $\downarrow$ & $\varnothing$ & $\uparrow$ & $\uparrow$ \\
\hline
\end{tabular}

Table 4: The impact of asset returns on $\bar{q}$ with $\gamma>2$

Assuming $f(\cdot)>0$ and $\gamma>2$ hold, using the fact of Table 4 , I can have that there exist $\epsilon>0$ and $\mathcal{R}>\rho_{1} \rho_{2}$ such that

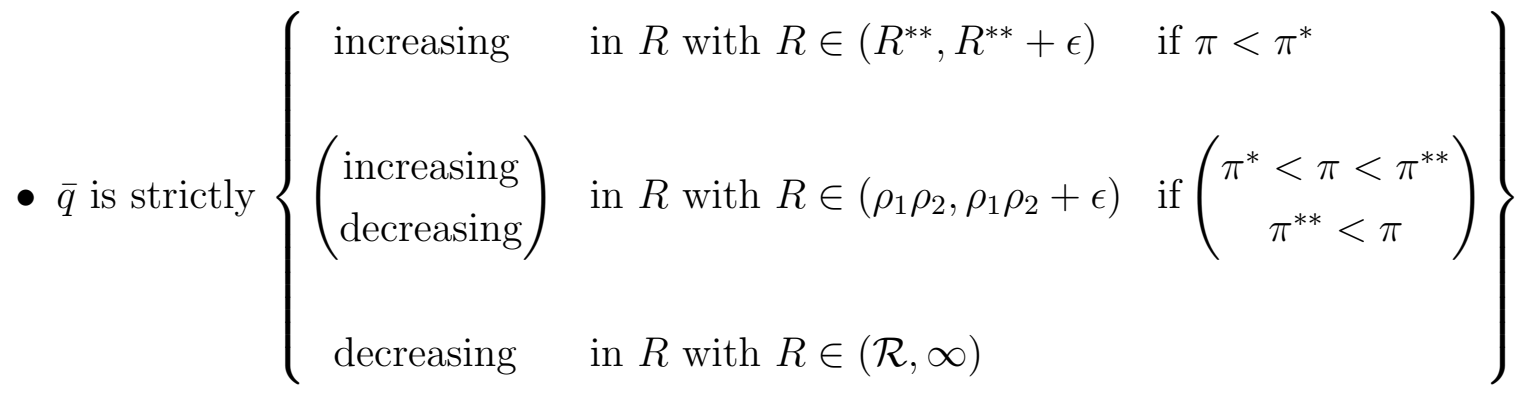

- $\bar{q}$ is $\left\{\begin{array}{cl}\left(\begin{array}{c}\text { increasing } \\ \text { constant }\end{array}\right) & \text { in } r \text { with } r \in(0, \epsilon) \text { if }\{q \mid h(q)=0 ; q \in(0,1)\}\left(\begin{array}{l}= \\ \neq\end{array}\right) \emptyset \\ \text { strictly decreasing } & \text { in } r \text { with } r \in\left(r^{*}, \min \left\{r^{* *}, \rho_{1}\right\}\right)\end{array}\right\}$

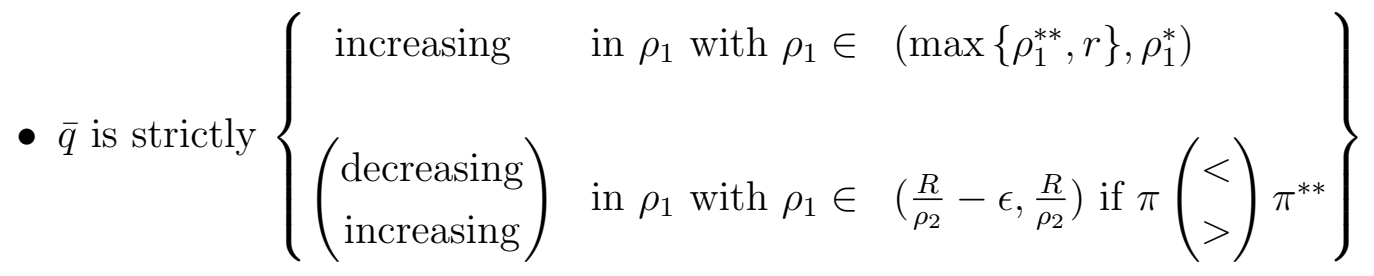

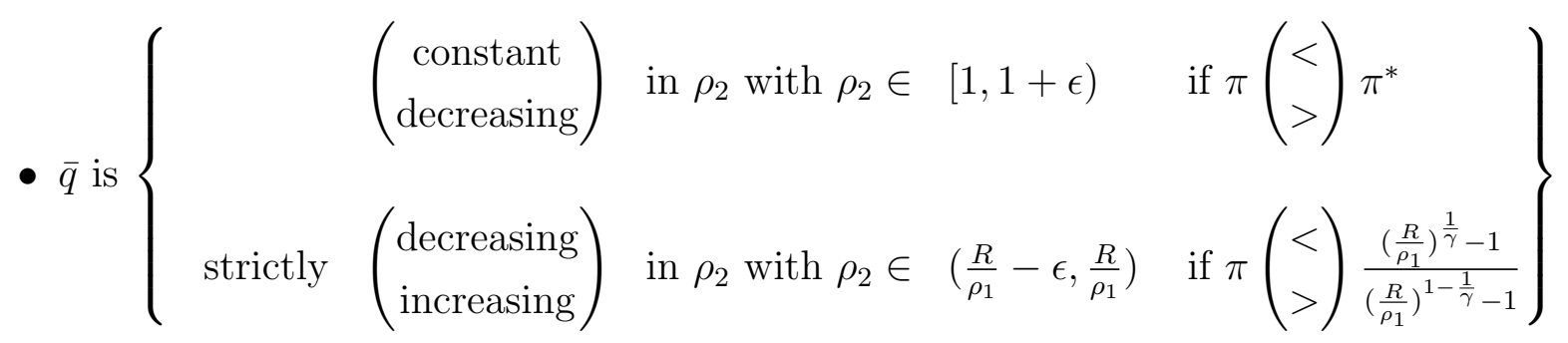

where $\pi^{* *}=\frac{\rho_{2}^{\frac{1}{\gamma}}-1}{\rho_{2}^{1-\frac{1}{\gamma}}-1}$. 


\section{E A General Definition of Financial Fragility}

I used a particular definition of fragility in the main text for simplicity; here I show that the same results are obtained if I use a more general definition. Suppose Definition 1 is replaced with the following.

Definition 3. A banking system is said to be fragile if there exists an equilibrium strategy profile with $y_{i}(1, \beta)=0$ for a positive measure of depositors; otherwise the banking system is said to be stable.

The following result shows that focusing on equation (4) is also necessary and sufficient for determining whether an economy is fragile under this more general definition.

Proposition 7. The economy is fragile if and only if (4) holds.

Proof. The proof of this proposition is divided into three steps as follows.

Step (i): $c_{1}^{*} \geq c_{2 \beta}^{*}$ is a sufficient condition for the economy to be fragile based on the discussion in the text. What remains to be proven is that is this condition is also necessary for fragility to arise.

Step (ii): Let $\tilde{y}$ be any such strategy profile with $\hat{\pi}_{\beta}<\pi$ and let $\tilde{\mathcal{A}}$ denote the allocation generated by the best response of the bank to this profile. This allocation is characterized by equations (10)-(18). If there is an equilibrium in which depositors follow $\tilde{y}$, it must be the case that $\tilde{c}_{1}=\tilde{c}_{2 \beta}$ holds. Suppose that $\tilde{c}_{1}>\tilde{c}_{2 \beta}$, which implies that all patient depositors with $i \leq \pi$ prefer to withdraw. However, the strategy profile $\tilde{y}$ proposes that the fraction of remaining depositors who are impatient after $\pi$ withdrawals have been made is less than $\pi$. Hence, there is an equilibrium in which depositors follow $\tilde{y}$ as long as $\tilde{c}_{1}=\tilde{c}_{2 \beta}$. Therefore, I must show that $c_{1}^{*} \geq c_{2 \beta}^{*}$ holds whenever $\tilde{c}_{1}=\tilde{c}_{2 \beta}$ holds for some $\tilde{y}$ in order to prove that $c_{1}^{*} \geq c_{2 \beta}^{*}$ is a necessary condition for fragility to arise.

- If the best-response allocation lies in Case I: from Appendix A, I see that $\frac{\tilde{c}_{1}}{\tilde{c}_{2 \beta}}$ is strictly increasing in $\hat{\pi}_{\beta}$;

- If the best-response allocation lies in Case II: from Appendix A, I see that $\frac{\tilde{c}_{1}}{\tilde{c}_{2 \beta}}$ is independent of $\hat{\pi}_{\beta}$;

- If the best-response allocation lies in Case III: the impact of $\hat{\pi}_{\beta}$ on $\frac{\tilde{c}_{1}}{\tilde{c}_{2 \beta}}$ and the nature of $\frac{\tilde{c}_{1}}{\tilde{c}_{2 \beta}}$ are determined as follows 
- if $\rho_{2} \leq\left(\frac{R}{\rho_{1}}\right)^{\frac{1}{\gamma}}$, then I have that $\tilde{c}_{1}<\tilde{c}_{2 \beta}$;

- if $\left(\frac{R}{\rho_{1}}\right)^{\frac{1}{\gamma}}<\rho_{2}<\left(\frac{R}{r}\right)^{\frac{1}{\gamma}}$, then I see that

$$
\left\{\begin{array}{c}
\tilde{c}_{1}<\tilde{c}_{2 \beta} \\
\frac{\tilde{c}_{1}}{\tilde{c}_{2 \beta}} \text { is strictly increasing in } \hat{\pi}_{\beta}
\end{array}\right\} \text { as } q \in\left\{\begin{array}{c}
(q u \underline{q}) \\
(\underline{q}, 1)
\end{array}\right\} \text {, where } \underline{q}=\frac{\frac{R}{\rho_{1}}-\rho_{2}}{\rho_{2}^{\gamma}-\rho_{2}} ;
$$

- if $\left(\frac{R}{r}\right)^{\frac{1}{\gamma}} \leq \rho_{2}$, then I see that $\frac{\tilde{c}_{1}}{\tilde{c}_{2 \beta}}$ is strictly increasing in $\hat{\pi}_{\beta}$.

In addition, differentiating $q_{l}$ and $q_{u}$ with respective to $\hat{\pi}_{\beta}$, I have $q_{l}$ is strictly decreasing in $\hat{\pi}_{\beta}$ and

$$
q_{u} \text { is strictly }\left\{\begin{array}{l}
\text { increasing } \\
\text { decreasing }
\end{array}\right\} \text { in } \hat{\pi}_{\beta} \text { if } \rho_{2}\left\{\begin{array}{l}
< \\
>
\end{array}\right\}\left(\frac{R}{r}\right)^{\frac{1}{\gamma}}
$$

Now, I show how these preliminary results combine to establish the proposition.

- If $\rho_{2} \leq\left(\frac{R}{\rho_{1}}\right)^{\frac{1}{\gamma}}$, then the best-response allocation $\tilde{\mathcal{A}}$ with $\tilde{c}_{1}=\tilde{c}_{2 \beta}$ could be in Case I or Case II.

Recall that $q_{l}\left(q_{u}\right)$ is strictly decreasing (increasing) in $\hat{\pi}_{\beta}$, it is straightforward to show the economy cannot be in Case III as $\hat{\pi}_{\beta}$ increases. Since $\frac{\tilde{c}_{1}}{\tilde{c}_{2 \beta}}$ is strictly increasing (independent) in $\hat{\pi}_{\beta}$ in Case I (II), I have $\frac{c_{1}^{*}}{c_{2 \beta}^{*}} \geq \frac{\tilde{c}_{1}}{\tilde{c}_{2 \beta}}$.

- If $\left(\frac{R}{\rho_{1}}\right)^{\frac{1}{\gamma}}<\rho_{2}<\left(\frac{R}{r}\right)^{\frac{1}{\gamma}}$, then the best-response allocation $\tilde{\mathcal{A}}$ with $\tilde{c}_{1}=\tilde{c}_{2 \beta}$ could be in Case I, Case II, or $q \in(\underline{q}, 1)$ of Case III.

Similarly, as $\hat{\pi}_{\beta}$ increases, the economy would be in Case I, Case II, or $q \in(\underline{q}, 1)$ of Case III. In this scenario, $\frac{\tilde{c}_{1}}{\tilde{c}_{2 \beta}}$ is non-decreasing in $\hat{\pi}_{\beta}$, which implies that $\frac{c_{1}^{*}}{c_{2 \beta}^{*}} \geq \frac{\tilde{c}_{1}}{\tilde{c}_{2 \beta}}$.

- If $\left(\frac{R}{r}\right)^{\frac{1}{\gamma}} \leq \rho_{2}$, then the best-response allocation $\tilde{\mathcal{A}}$ with $\tilde{c}_{1}=\tilde{c}_{2 \beta}$ could be in Cases I, II, or III. Using the results above, I have $\frac{\tilde{c}_{1}}{\tilde{c}_{2 \beta}}$ is non-decreasing in $\hat{\pi}_{\beta}$, which implies that $\frac{c_{1}^{*}}{c_{2 \beta}^{*}} \geq \frac{\tilde{c}_{1}}{\tilde{c}_{2 \beta}}$.

Therefore, $\frac{\tilde{c}_{1}}{\tilde{c}_{2 \beta}}=1$ implies $\frac{c_{1}^{*}}{c_{2 \beta}^{*}} \geq 1$, as desired.

Step (iii): Now suppose that $c_{1}^{*}<c_{2 \beta}^{*}$ holds. Using Appendix A and the results from Step (ii), this inequality implies that $\bar{c}_{1}<\bar{c}_{2 \beta}$ for the profile $\bar{y}$ in which run never occurs. For the converse, note that if the economy is stable, it follows immediately that $c_{1}^{*}<c_{2 \beta}^{*}$ must hold and concludes the proof. 


\section{References}

[1] David Andolfatto, Ed Nosal, and Bruno Sultanum. Preventing bank runs. Federal Reserve Bank of Chicago Working Paper, 2014.

[2] Jefferson DP Bertolai, Ricardo de O Cavalcanti, and Paulo K Monteiro. Run theorems for low returns and large banks. Economic Theory, 57(2):223-252, 2014.

[3] Russell Cooper and Thomas W Ross. Bank runs: Liquidity costs and investment distortions. Journal of Monetary Economics, 41(1):27-38, 1998.

[4] Douglas W Diamond and Philip H Dybvig. Bank runs, deposit insurance, and liquidity. Journal of Political Economy, 91(3):401-419, 1983.

[5] Huberto M Ennis and Todd Keister. Bank runs and investment decisions revisited. Journal of Monetary Economics, 53(2):217-232, 2006.

[6] Huberto M Ennis and Todd Keister. Bank runs and institutions: The perils of intervention. American Economic Review, 99(4):1588-1607, 2009.

[7] Huberto M Ennis and Todd Keister. Banking panics and policy responses. Journal of Monetary Economics, 57(4):404-419, 2010.

[8] Todd Keister. Bailouts and financial fragility. Review of Economics Studies (forthcoming), 2015.

[9] James Peck and Karl Shell. Equilibrium bank runs. Journal of Political Economy, 111(1):103-123, 2003.

[10] Bruno Sultanum. Optimal Diamond-Dybvig mechanism in large economies with aggregate uncertainty. Journal of Economic Dynamics and Control, 40:95-102, 2014.

[11] Neil Wallace. Another attempt to explain an illiquid banking system: The Diamond and Dybvig model with sequential service taken seriously. Federal Reserve Bank of Minneapolis Quarterly Review, 12(4):3-16, 1988.

[12] Neil Wallace. A banking model in which partial suspension is best. Federal Reserve Bank of Minneapolis Quarterly Review, 14(4):11-23, 1990. 